\title{
Electronic structure of the $\mathrm{GaSb}(001)$ surface
}

\author{
J. Olde, K.-M. Behrens, H.-P. Barnscheidt, ${ }^{*}$ R. Manzke, and M. Skibowski \\ Institut für Experimentalphysik, Universität Kiel, Olshausenstrasse 40, D-2300 Kiel 1, Federal Republic of Germany \\ J. Henk and W. Schattke \\ Institut für Theoretische Physik und Sternwarte, Universität Kiel, Olshausenstrasse 40, D-2300 Kiel 1, Federal Republic of Germany
}

(Received 6 February 1991)

\begin{abstract}
The $\mathrm{GaSb}(001)$ surface prepared by ion bombardment and annealing is investigated by angle-resolved photoemission spectroscopy using $\mathrm{He}$ I and synchrotron radiation with photon energies between 9 and $34 \mathrm{eV}$. The band structure is traced along the $\Gamma-\Delta-X$ line normal to the surface and along lines of high symmetry of the surface Brillouin zone by measuring the energy-distribution curves and constantinitial-state and constant-final-state spectra. The results are compared with theoretical bulk band structures; in particular, the normal-emission data were analyzed by using structure plots, and in this way the most dispersing features in normal emission could be explained. Furthermore, several surface states or resonances are found showing the dispersion of a reconstructed surface. The spectral features of constant-initial-state spectra reflect mainly the band edges of the unoccupied bulk band structure and are probably associated with emissions from a surface state or backfolded bulk bands close to the $X_{3}$ critical-point energy. The decay of the $\mathrm{Ga} 3 d$ core exciton is observed in constant-final-state spectra, indicating the existence of an unoccupied surface state at the Ga-rich surface.
\end{abstract}

\section{INTRODUCTION}

In recent years, III-V semiconductors have gained technological interest, especially concerning the fabrication of optoelectronical devices such as semiconductor lasers or photodetectors. In order to understand the electronic properties of these materials, it is of great importance to study the electronic structure of bulk and surface. Their knowledge can be used to develop reliable models of interfaces between semiconductors and metals or between different semiconductor. Until now GaAs has been the best studied compound semiconductor. Not only the cleavable (110) surface, but also the other lowindexed crystal planes as (001), (111), and ( $\overline{1} \overline{1} \overline{1})$ have become subject to bandstructure investigations by means of angle-resolved photoemission spectroscopy (ARPES), e.g., Refs. 1-9. Also, calculations of the surface band structure based on different surface structure models are available for the (110), (111), and ( $\overline{1} \overline{1} \overline{1})$ surfaces $^{10-14}$ and to some extent for simplified models of the (001) surface. ${ }^{5}$

For GaSb, there are few experimental or theoretical investigations of the electronic band structure, and the work is concentrated on the cleavable (110) plane. ${ }^{11,15-17}$ Only in a very recent paper by Franklin et al. ${ }^{18}$ the $\mathrm{GaSb}(001)-(1 \times 3)$ surface prepared by molecular-beam epitaxy (MBE) was investigated by ARPES with energydistribution curves (EDC's) in normal emission, interpreting the main spectral features. It is desirable to obtain a thorough understanding of the electronic structure of this plane basing not only on the strong spectral features but also incorporating the weaker, still distinct ones analyzed by several different modes of ARPES and separately considering also the influence of the final states beyond the free-electron model.

In this work we present our investigations of the
$\mathrm{GaSb}(001)$ surface prepared by ion bombardment and annealing, which we have performed in order to get further information about the bulk band structure including higher conduction bands, as well as to evaluate an electronic surface band structure, which may help to develop or to improve surface structure models as given in Ref. 18. From normal-emission energy-distribution curves, an experimental valence-band structure along the $\Gamma-\Delta-X$ line of the bulk was derived and compared to theoretical bulk band calculations also by means of structure plots. Constant-final-state (CFS) spectroscopy was performed in order to confirm the results obtained from the EDC's. Furthermore, the electronic states were traced along high-symmetry lines of the ideal surface. A separation of bulk and surface states or resonances became possible in most cases by calculating the positions of bulk-derived emissions in the frame of the free-electron final-state approximation and assuming $\mathbf{k}$ conservation. $\mathrm{Up}$ to seven surface-derived bands were found. For a refined analysis, final states calculated by the empirical pseudopotential method (EPM) were also used. Moreover, we applied constant-initial-state (CIS) spectroscopy to obtain information about the origin of the emissions close to the $X_{3}$ critical-point energy appearing in nearly all normal- and off-normal-emission spectra. The results are shown to be in good agreement with the EPM band structure. Finally, depending on the polarization conditions of the incident light, in normal-emission CFS spectra the excitation of the Ga3d core exciton was observed, which indicated the presence of an unoccupied surface state close to the conduction-band minimum (CBM).

\section{EXPERIMENT}

The $\mathrm{GaSb}(001)$ samples used here were cut from a (001)-oriented $p$-type doped GaSb single crystal and pol- 
ished with diamond polishing compound down to 0.25 $\mu \mathrm{m}$. After degreasing in trichlorethylene, acetone, and methanol, they were mounted to molybdenum sample holders by liquid indium. Then the samples were brought into ultrahigh-vacuum (UHV) preparation and investigation systems: The surfaces investigated by low-energy electron diffraction (LEED) and reflection high-energy electron diffraction (RHEED) were prepared in our laboratory in Kiel, while those investigated by ARPES were produced in a preparation chamber equipped with an ion gun and an electron-bombardment sample heater installed in our ARPES system at HASYLAB (Hamburg Synchrotron Radiation Laboratory). All samples investigated here were cleaned by $800-\mathrm{eV}$ argon ions for $3 \mathrm{~h}$ and then annealed at about $490^{\circ} \mathrm{C}$ for $15 \mathrm{~min}$.

In Fig. 1 the resulting RHEED [Figs. 1(a) and 1(b)] and LEED [Fig. 1(d)] pictures of the surface are given: They show a $(2 \times 3)$ or $c(2 \times 6)$ reconstruction, where the $\frac{1}{3}$.

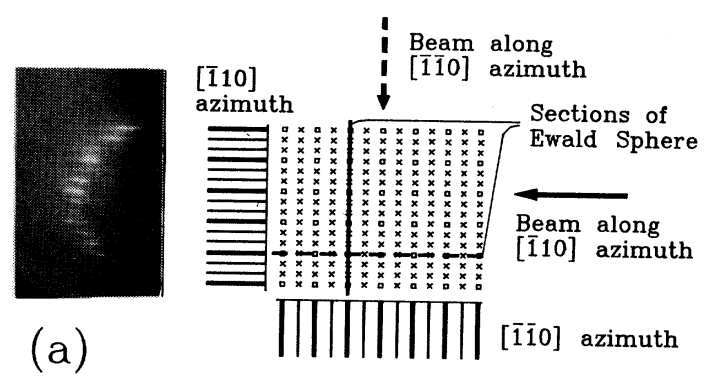

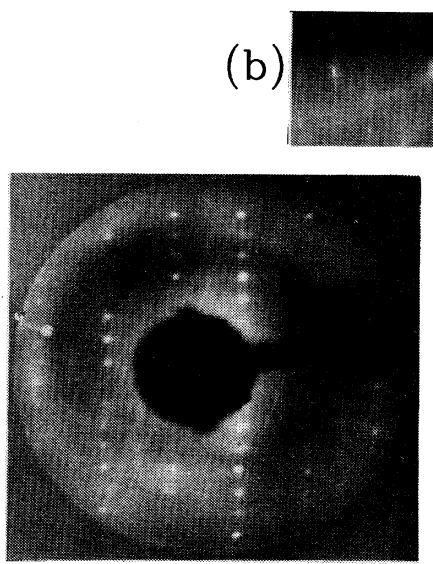

(d)
FIG. 1. (a) and (b): RHEED pattern of $\mathrm{GaSb}(001)-(2 \times 3)$ at $8-\mathrm{keV}$ kinetic energy. In (a) the [ $\overline{1} 10]$ azimuth is shown, (b) is the same for the $[\overline{1} 10]$ azimuth. In (c) the reciprocal lattice is sketched together with the incident beam direction, sections of the Ewald sphere, and the resulting RHEED pictures. The reciprocal-lattice rods from the ideal $(1 \times 1)$ surface are plotted as squares or heavy lines, those from the reconstructed surface as crosses or thin lines at the left and lower side of (c). (d) LEED pattern of the same reconstruction at 116-eV kinetic energy. In (e) the reciprocal lattice of $(2 \times 3)$ is shown together with the experimental results plotted as closed squares, closed circles or heavy lines: the $\frac{1}{2}$-order reflexes are blurred to lines. The hatched area shows the position of the electron gun. order streaks or points between the integer-order streaks or points are distinct while the $\frac{1}{2}$-order streaks or points are very weak and blurred. Therefore, it is not possible to distinguish between the two reconstruction types, which differ only by the position of the half-order reflexes. In Figs. 1(c) and 1(e) we have sketched the theoretically expected and the experimentally observed pattern for a $(2 \times 3)$ reconstruction. This behavior is quite similar to that observed at the $\operatorname{GaAs}(001)-(2 \times 4)$ or $c(2 \times 8)$ surface, where the blurred streaks in the diffraction patterns can be explained by some disorder of the surface structure. ${ }^{19}$ The same may be true in the case of $\mathrm{GaSb}(001)$. In Ref. 18, this fact is confirmed for MBE-prepared samples by scanning tunneling microscopy (STM) images. Here we remark that, using MBE systems, some authors distinguish between $c(2 \times 6)$, $(2 \times 3)$ and also $c(2 \times 10)$ and $(1 \times 3)$ reconstructions depending on preparation conditions such as flux ratio and sample temperatures. ${ }^{18,20,21}$ In our ion-bombardment and annealing (IBA) experiments the observed diffraction pattern was nearly independent of the annealing temperature in the range from about $350^{\circ} \mathrm{C}$ to about $500^{\circ} \mathrm{C}$.

In order to investigate the samples prepared at HASYLAB by ARPES, they were transferred by magnetic driven transfer rods into the spectrometer chamber equipped with a $180^{\circ}$ spherical electron energy analyzer mounted on a two-axes goniometer. The angular resolution is $\pm 0.5^{\circ}$ for polar and azimuthal angles and the energy resolution was chosen as $130 \mathrm{meV}$ full width at half maximum (FWHM) for most spectra. The electrons were excited by a He I discharge lamp or by synchrotron radiation from the DORIS II storage ring. In the latter case, the photon energy was varied in the range from 9 to 34 $\mathrm{eV}$ with a wavelength resolution of $2.3 \AA$ and a degree of linear polarization from $80 \%$ to $98 \%$ depending on photon energy. With the sample orientation chosen here, the surface normal, a $\langle 110\rangle$ direction, and the mean vector potential $\mathbf{A}$ of the incident synchrotron light were in the same plane, $A$ at an angle of about $45^{\circ}$ with the surface normal.

\section{RESULTS AND DISCUSSION}

\section{A. Normal emission}

Energy-distribution curves have been taken along the $\Gamma-\Delta-X$ direction of the bulk and along lines of high symmetry of the ideal $(1 \times 1)$ surface Brillouin zone (SBZ) (cf. Fig. 2). We start with a discussion of the normalemission spectra. Figure 3 shows angle-resolved EDC's in normal emission with photon energies in the range from 9 to $34 \mathrm{eV}$. The marked positions of the emissions were determined by a fit procedure after background subtraction. The features marked $B-E$ and $G-J$ and connected by lines exhibit clear dispersion of their binding energies with photon energy and are therefore attributed to emissions from bulk bands. It will be shown below that also the less-pronounced weakly dispersing states $\boldsymbol{A}$ and $F$ are very likely attributed to bulk emissions. The energy positions of states with no or almost no dispersion are assigned by the lowercase letters $a-g$ in the upper part of Fig. 3 (if the states are slightly dispersing, a mean 

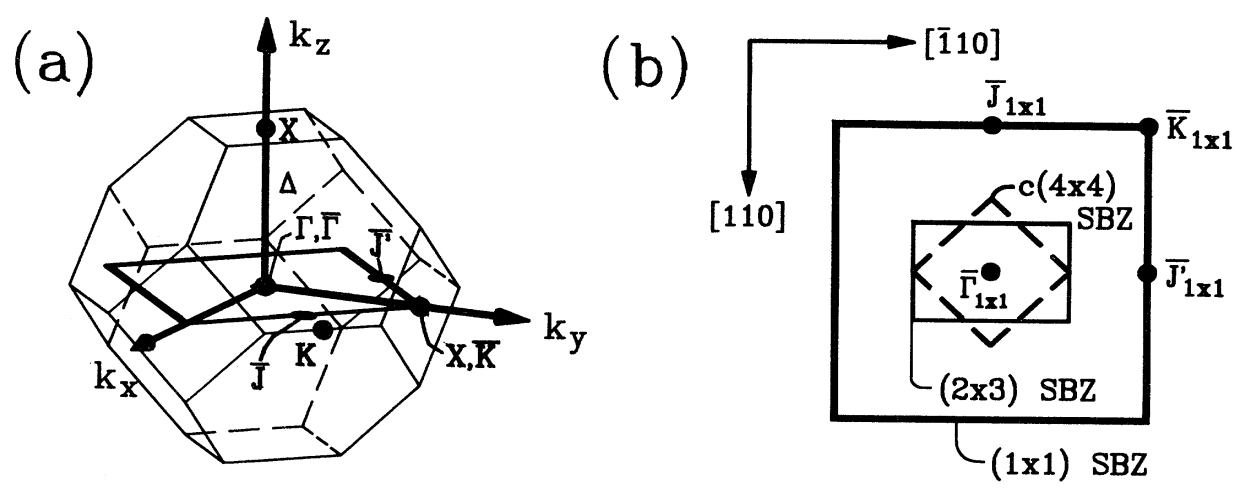

FIG. 2. (a) $(1 \times 1)$ surface Brillouin zone (SBZ) inserted into the bulk Brillouin zone. (b) gives a top view of the $(1 \times 1),(2 \times 3)$, and $c(4 \times 4) \mathrm{SBZ}$. For the $c(2 \times 6) \mathrm{SBZ}$ see Fig. 17.

value of the energy is chosen), but only the features $a, b$, $f$, and $g$ are observed over a wide range of photon energies. The peaks are partly superimposed by the dispersing structures. Some of these features will be shown to be due to surface states or resonances by tracing them also

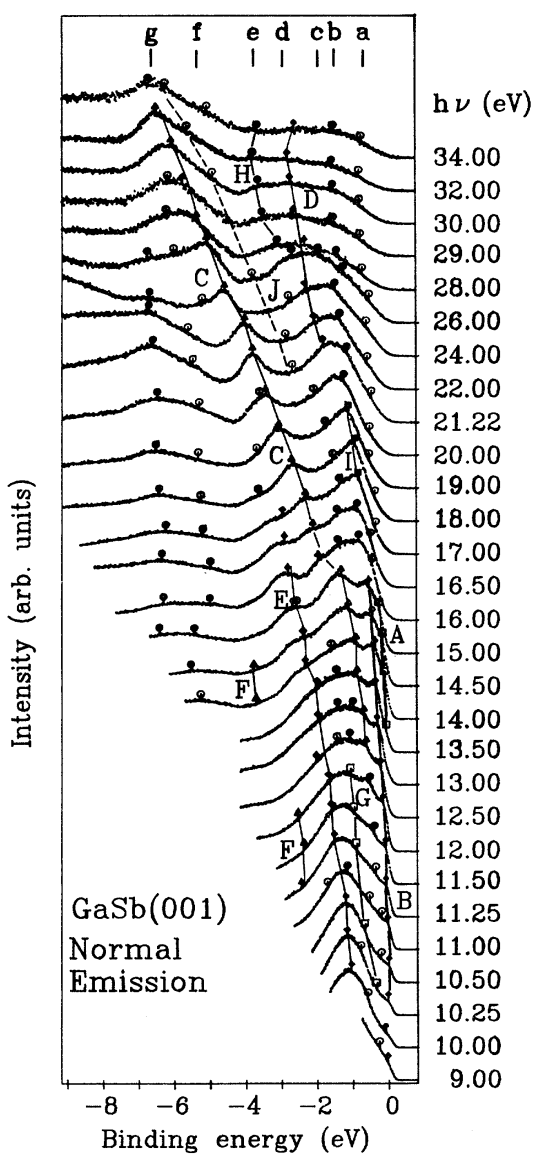

FIG. 3. Angle-resolved photoemission spectra taken in normal emission for different photon energies. The binding energy is related to the valence-band maximum. The structures labeled $A-J$ are due to emissions from dispersive bulk bands. The bars labeled $a-g$ in the upper part of the plot mark the energy positions of nondispersive emissions. along the high-symmetry lines of the surface in offnormal measurements (see Sec. III B).

The normal-emission EDC's can be directly compared to those shown in Ref. 18 for lower photon energies, noticing that in our spectra the zero of energy is the valence-band maximum (VBM), the Fermi level lying $0.11 \mathrm{eV}$ above the VBM. The main dispersing structures have nearly the same energy positions; however, the shape of the spectra is quite different. This fact may be caused by different polarization conditions, but can also be attributed to different surface reconstructions according to emissions from surface states.

\section{Interpretation of EDC's using free-electron-like final states}

For a first evaluation, a valence-band structure $E\left(k_{\perp}\right)$ along the $\Gamma-\Delta-X$ line can be obtained from the spectra by using the well-known equation for the determination of the wave-vector component of electrons perpendicular to the surface in the first Brillouin zone (BZ)

$$
k_{\perp}=\left[\left(2 m / \hbar^{2}\right)\left(E_{\mathrm{kin}}+\left|V_{0}\right|\right)-g_{\|}^{2}\right]^{1 / 2}-G_{\perp},
$$

which is valid in the free-electron-like final-state approximation. Here $E_{\text {kin }}$ is the kinetic energy of the emissions, $\left|V_{0}\right|$ is the inner potential related to the vacuum level, and $G_{\perp}$ is the normal component of a reciprocal bulk lattice vector used for reduction into the first BZ. $g_{\|}$is any reciprocal surface lattice vector, i.e., the parallel component of a reciprocal bulk lattice vector or a reciprocallattice vector of the ideal or reconstructed surface or a combination of both. In Fig. 4 the resulting experimental band structure is shown using $\mathbf{g}_{\|}=0$ and $G_{\perp}=4 \pi / a$, where $a=6.0959 \AA$ is the lattice constant of GaSb. The bands are marked by the same symbols and letters as in Fig. 3. Here all peaks and shoulders observed in the spectra are shown, not only the subset of strong dispersing features. The solid lines labeled 1-3 show for comparison a bulk band structure along the $\Gamma-\Delta-X$ line calculated by the empirical tight-binding method (ETBM) (Ref. 22) and fitted to that of Chelikowsky and Cohen. ${ }^{15}$ The VBM is determined by the emission of $B$ in the 11eV spectrum, i.e., the minimum energy of a dispersing 


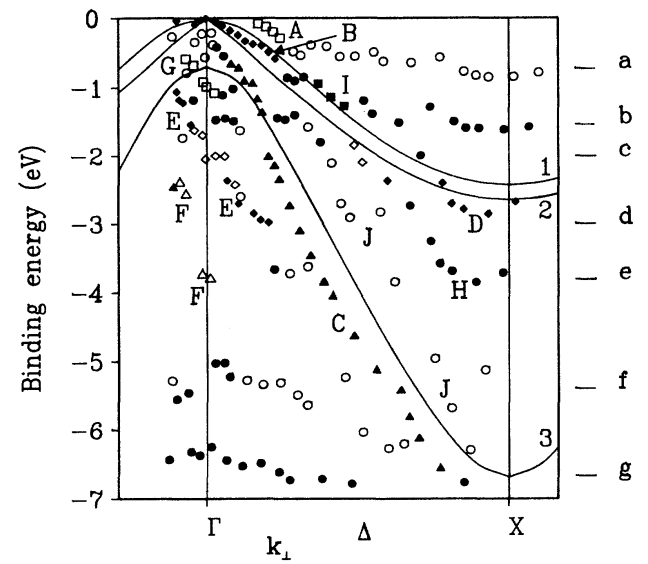

FIG. 4. Experimental band structure along the $\Gamma-\Delta-X$ direction of the bulk obtained from the spectra in Fig. 3 by Eq. (1). The solid lines labeled 1-3 show the theoretical band structure from Chelikowsky and Cohen (Ref. 15). Experimental structures related to the $\Gamma_{8} X_{7}$ band (labeled 1) are marked by squares, whereas rhombs denote emissions from the $\Gamma_{8} X_{6}$ band (labeled 2) and triangles those from the $\Gamma_{7} X_{6}$ band (labeled 3). All other emissions are plotted as circles. Closed symbols mark strong, open symbols weaker structures.

and therefore bulk-related band, and $\left|V_{0}\right|$ is chosen as 11 $\mathrm{eV}$ in order to obtain the best symmetry of the emissions of $B$ around the $\Gamma$ point. This value is significantly lower than that known from the $\mathrm{GaSb}(110)$ surface, ${ }^{23}$ as in the case of $\mathrm{GaAs},{ }^{7}$ showing that $V_{0}$ is not a unique physical quantity of $\mathrm{GaSb}$ but is mainly a fit parameter in order to fit in a simple way parabola-shaped bands to the conduction-band structure and to consider some aspects due to the photoemission process such as, e.g., broadening and shifts of the band structures.

Some of the dispersive structures may be related to theoretical bands: The emissions of $\mathrm{C}$ are due to valence band 3 (the $\Gamma_{7} X_{6}$ band), even if their binding energy is higher for larger values of $k_{\perp}$, i.e., $k_{\perp} \geq \frac{1}{4} k_{\Gamma X}$, but only the $\Gamma_{7} X_{6}$ band shows a dispersion of about $5.5 \mathrm{eV}$ as is observed experimentally. $B$ is related to valence band 1 or 2 (for a more precise determination see below), the weak structure $A$ possibly to the $\Gamma_{8} X_{7}$ band 1. Feature $D$ results from band 2 , the $\Gamma_{8} X_{6}$ band. The peaks $I$, which are very pronounced in contrast to those of $A$, may also be related to valence band 1 , but this is not very clear, since the emissions are close in energy to state $b$. The other dispersing features are not explained in this picture, which is adapted to primary cone emissions only, i.e., $\mathbf{g}_{\|}=\mathbf{0}$ and $G_{\perp}$ is a reciprocal bulk lattice vector itself. The non or weakly dispersing emissions $a-g$ are far away from bulk bands and are very likely due to surface states or resonances. However, the structures $e$ and $g$ are close in energy to the critical point $\Sigma_{1 \mathrm{~min}}$ at $-3.6 \mathrm{eV}$ and to the $X_{3}$ point at $-6.8 \mathrm{eV}$ following Ref. 15. In Ref. 18, the agreement between theory and experiment is slightly better. This is mainly due to the larger value of $\left|V_{0}\right|$ : in Ref. $18,\left|V_{0}\right|$ is taken as $7.2 \mathrm{eV}$ related to $E_{F}$. With $E_{F}-E_{\mathrm{VBM}}=0.25 \mathrm{eV}$ from Ref. 18 and $U_{\mathrm{th}} \approx 5.3 \mathrm{eV}$ from our measurements, this would result in a value of about $12.3 \mathrm{eV}$ of the inner potential related to the vacuum level. With this value, the symmetry of state $B$ around $\Gamma$ would become worse in our results; furthermore, the freeelectron final-state band structure with $\left|V_{0}\right|=11 \mathrm{eV}$ is in quite good agreement with final states calculated by the pseudopotential method.

For a further analysis of the dispersing structures we now turn to the method of structure plots described in detail elsewhere. ${ }^{2,7}$ In the following plots the theoretical curves show the $\mathbf{k}$-conserving transitions, neglecting matrix elements, between a valence-band structure calculated by ETBM including spin-orbit splitting ${ }^{22}$ and fitted to that of Chelikowsky and Cohen, ${ }^{15}$ and free-electron-like final states described by

$$
E_{F}=\left(\hbar^{2} / 2 m\right)\left[\left(k_{\perp}+G_{\perp}\right)^{2}+\mathbf{g}_{\|}^{2}\right]-\left|E_{0}\right|,
$$

where $\left|E_{0}\right|$ is the inner energy related to the VBM. With an inner potential of $\left|V_{0}\right|=11 \mathrm{eV}$ (see above) and an experimental photoelectron threshold $U_{\text {th }}$ of $5.3 \mathrm{eV}$ resulting from our measurements, we have $\left|E_{0}\right|=5.7 \mathrm{eV}$. The free-electron bands are only an approximation of the final states involved in the photoemission process, but may be used to describe the fundamental characteristics of the spectral features observed in experiment. In the next section, EPM final states will be used for a more detailed analysis.

At first we discuss a structure plot using the valenceand conduction-band structure along the $\Gamma-\Delta-X$ line, i.e., without backfolding with reciprocal surface lattice vectors. In Eq. (2) only such pairs $\left(G_{1}, \mathbf{g}_{\|}\right)$are used, for which $\mathbf{G}_{\perp}+\mathbf{g}_{\|}$is a reciprocal bulk lattice vector. The free-electron-like band structure is plotted in Fig. 5 as dashed lines, together with a pseudopotential conduction-band calculation discussed later. In Fig. 6 the resulting structure plot is shown together with the experimental values. Solid curves are due to transitions from the $\Gamma_{8} X_{7}$ valence band (band 1 in Fig. 4), dashed curves are due to the $\Gamma_{8} X_{6}$ band (band 2), and dashedbroken curves due to the $\Gamma_{7} X_{6}$ band (band 3 ). The experimentally observed structures are labeled with the same symbols as in Fig. 4. The energy positions of nondispersing or nearly nondispersing structures are marked by bars at the right side of the plot. The emissions $A-D$ and $I$ are lying relatively close to the theoretical structure curves with final states described by $\mathbf{g}_{\|}=0$ and $G_{\perp}=4 \pi / a$. This is in accordance with Fig. 4, where only this final state was used. In addition, one finds a correspondence of features $E, F$, and $G$ to theoretical structure curves: the peaks of $G$ are caused by transitions from the $\Gamma_{8} X_{7}$ band into all final states described by $|\mathbf{G}|=k_{\Gamma \Lambda L \Lambda \Gamma}$ with $G_{1}=2 \pi / a$ and $G_{\|}=2^{1 / 2} 2 \pi / a$, which are commonly degenerate along $\Gamma-\Delta-X . F$ is the same for the $\Gamma_{7} X_{6}$ valence band. In $E$ the peaks at lower photon energies up to about $14 \mathrm{eV}$ are also emissions from those final states, but at higher photon energies the electrons seems to arise mainly from the conduction bands with $|\mathbf{G}|=k_{\Gamma \Delta X \Delta \Gamma}$ and $G_{\perp}=0$.

Up to now it can be concluded that the most dispersing features in the spectra can be quite well described by 


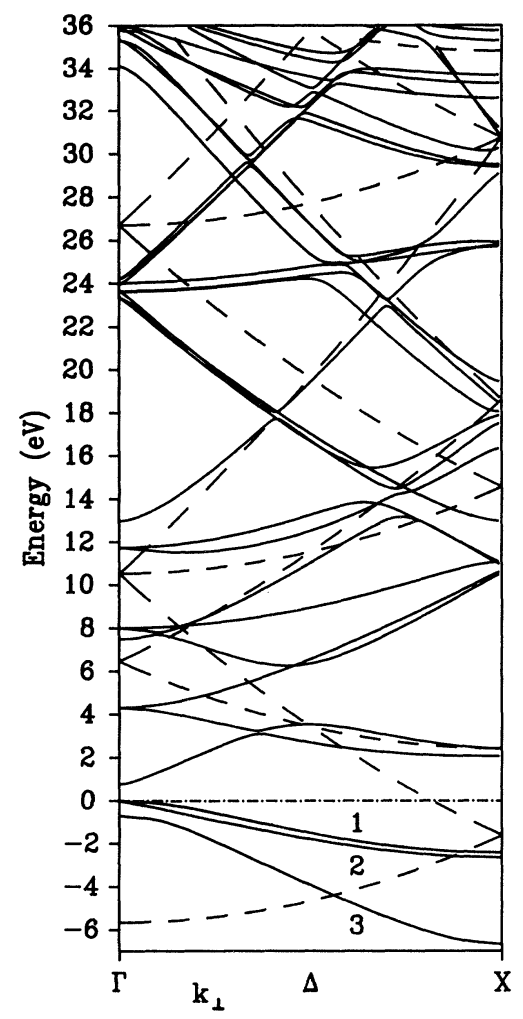

FIG. 5. Theoretical band structure along the $\Gamma-\Delta-X$ line of $\mathrm{GaSb}$ used in this paper. The valence bands are calculated by the empirical tight-binding method, the $\Gamma_{8} X_{7}$ band is labeled 1, the $\Gamma_{8} X_{6}$ band is labeled 2, and the $\Gamma_{7} X_{6}$ band is labeled 3. The conduction bands are calculated by an empirical pseudopotential. Also shown by dashed lines are the free-electron-like bands with $\left|V_{0}\right|=11 \mathrm{eV}$ as used in Figs. 6 and 7. transitions from the valence bands to different final state bands of the free-electron-like parabola, with $\mathbf{g}_{\|}+\mathbf{G}_{\perp}$ being a reciprocal bulk lattice vector. In order to explain also the dispersing features $J$ and $H$, we have investigated the effect of backfolding with reciprocal surface lattice vectors on the transitions between bulk bands. Due to the great number of reciprocal surface lattice vectors different from reciprocal bulk lattice vectors in higherorder reconstructions and to the lifting of degeneracy of the bulk bands along lines apart from high-symmetry lines of the bulk, the resulting structure plots can become very congested, and without further knowledge of selection rules nearly all experimentally observed emissions can be explained by some structure curves. Therefore, here we will give only two examples for a possible explanation of the structures $J$ and $H$.

Since the parts of structure $H$ with higher binding energies are close in energy to the $\Sigma_{1 \text { min }}$ critical point it seems to be legitimate to look for a reciprocal surface lattice vector, which folds back the $\Sigma_{1 \text { min }}$ point lying at about $\mathbf{k}_{\|}=\frac{2}{3} \mathbf{k}_{\Gamma K X}$ onto the $\Gamma-\Delta-X$ line. In the $(2 \times 3)$ or $c(2 \times 6)$ reconstruction observed by LEED and RHEED for this surface, there are vectors $\mathbf{g}_{\| 2 \times 3}=\frac{2}{3} \mathbf{k}_{\Gamma K X}$ (cf. Fig. 2 , noting that $\bar{\Gamma} \bar{J} \bar{\Gamma} \hat{=} \Gamma K X)$, i.e., of the desired type. In Fig. 7 the structure curves are shown, which are obtained by projecting the valence-band structure along $\mathbf{g}_{\| 2 \times 3}+\mathbf{k}_{\perp}$, calculated by ETBM, ${ }^{22}$ onto the $\Gamma-\Delta-X$ line, i.e., $E_{b}\left(g_{\|}, k_{\perp}\right)$ also describes electronic states along the $\Gamma-\Delta-X$ line, and with the same conduction bands as used in Fig. 6 , i.e., the conduction bands of the $\Gamma-\Delta-X$ line shown in Fig. 5 are used. The most striking fact is the good correspondence between the emissions of $H$ and theoretical curves from the second pair of valence bands (i.e., the bands belonging to the $\Gamma_{8} X_{6}$ band on the $\Gamma-\Delta-X$ line), which contain the $\Sigma_{1 \text { min }}$ point. For higher photon ener-

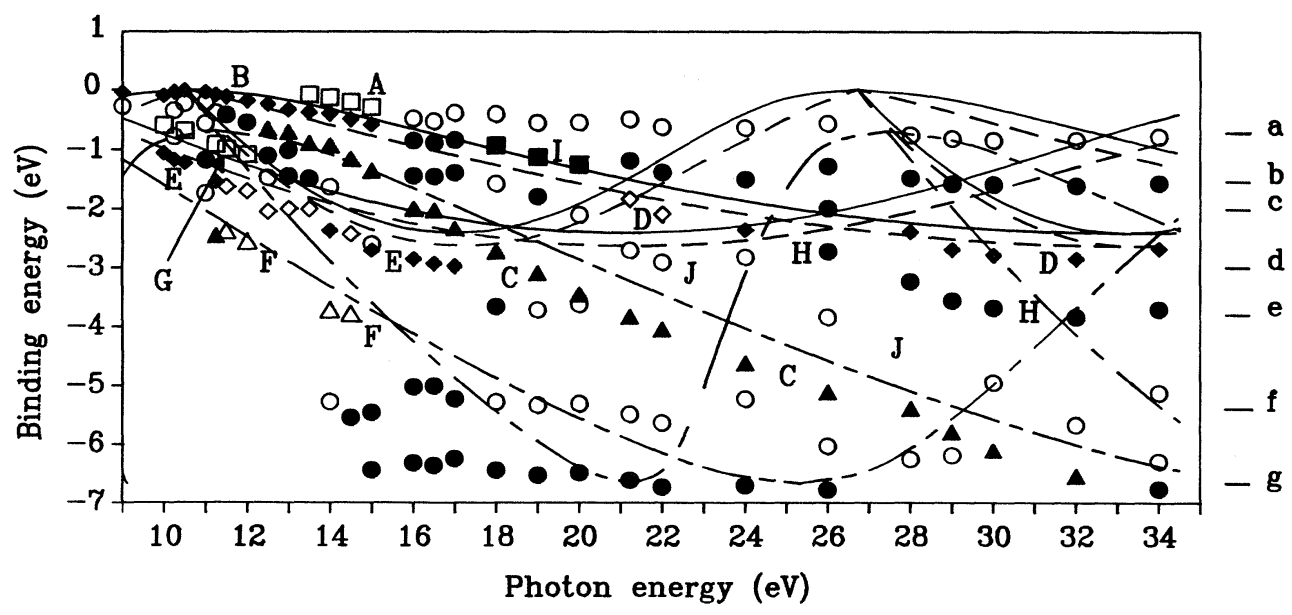

FIG. 6. Structure plot from the experimental data. Emissions marked by squares are due to the $\Gamma_{8} X_{7}$ band, those marked by rhombs to the $\Gamma_{8} X_{6}$ band, and those by triangles to the $\Gamma_{7} X_{6}$ band; all other structures are plotted as circles. Closed symbols indicate strong, open symbols weaker structures. Positions of nondispersive structures are marked by horizontal bars and assigned by the same symbols as in Fig. 3. The transitions are assigned by the same letters as in Fig. 3. The lines present theoretical structure curves; transitions from the $\Gamma_{8} X_{7}$ band into free-electron-like final states (solid lines), from the $\Gamma_{8} X_{6}$ band (dashed lines), and from the $\Gamma_{7} X_{6}$ band (dashed-broken lines). 


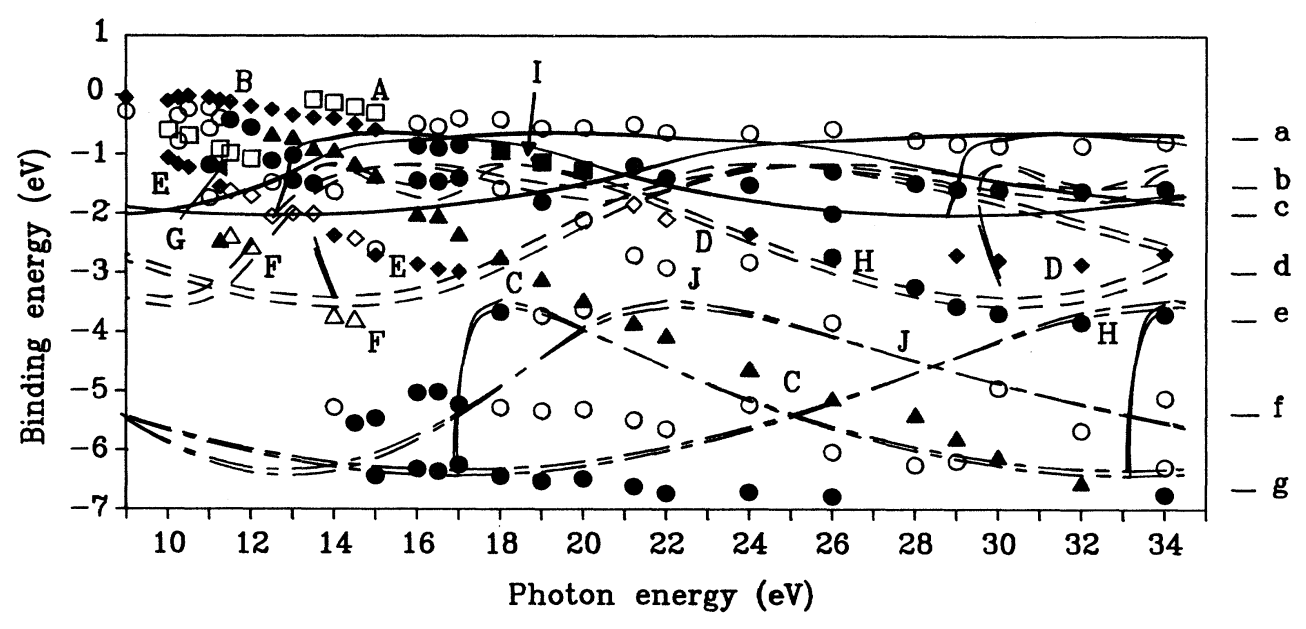

FIG. 7. Same as in Fig. 6, but with backfolding effects. The reciprocal-lattice vector used here is $\mathbf{g}_{\|}=\frac{2}{3} \mathbf{k}_{\bar{\Gamma} \bar{J} \bar{\Gamma}}$ of the reconstructed surface. For details see text.

gies, such as about $32 \mathrm{eV}$, the peaks are explained by transitions from the third pair of bulk valence bands. In the photon-energy range of above $25 \mathrm{eV}$, the feature $J$ is also close to a curve from those valence bands. However, a correspondence between feature $J$ and this curve is questionable, since the other observed emissions from this valence band, the structures $C$ and $F$, were lying below the theoretically predicted curves. Furthermore, there are theoretical structure curves that are quite close to the emissions of $a$ and $b$ showing also nearly no dispersion over a wide range of photon energies. It will be shown in Sec. III B that, nevertheless, features $a$ and $b$ are very likely surface derived according to their dispersion along high-symmetry lines of the surface.

As will be described below, the electronic structure shows not a very distinct $(2 \times 3)$ or $c(2 \times 6)$ behavior in off-normal emission, and the periodicity of some surface states is quite similar to that expected for a $c(4 \times 4)$ reconstructed surface. In the latter case, the influence of the surface reconstruction onto bulk transitions should be governed by reciprocal-c $(4 \times 4)$-lattice vectors, and an explanation of $H$ as discussed above must be abandoned.

This problem can be overcome if backfolding with the vector $\mathbf{g}_{\| c(4 \times 4)}=\frac{1}{2} \mathbf{k}_{\Gamma \Delta X}$, which is a reciprocal-lattice vector of the $c(4 \times 4)$ reconstruction, is considered. The structure plot obtained from the transitions between the valence and conduction bands along the line $\mathbf{k}=\mathbf{g}_{\| c(4 \times 4)}+\mathbf{k}_{\perp}$ is quite difficult to survey due to the great number of structure curves and should be interpreted very guardedly. Nevertheless, most peaks of $H$ may be described by structure curves from the second pair of valence bands.

In summary, emission $H$ is in the reasonable agreement with structure curves obtained from backfolding with reciprocal surface lattice vectors. The correspondence of some other weaker features to theoretical curves in the structure plots may be fortuitous because of the large number of curves.

\section{Interpretation of EDC's using final states calculated by an empirical pseudopotential}

Though nearly all dispersing structures in the spectra are in principle explainable by transitions from the calculated valence bands used here into different branches of the free-electron final-state parabola, there are some problems remaining. The experimentally observed transitions from the $\Gamma_{7} X_{6}$ band (band 3 in Fig. 4) into the final-state branch with $\mathrm{g}_{\|}=0$ and $G_{\perp}=4 \pi / a$ (marked $C$ ) are lying at significantly higher binding energies for photon energies between about 15 and $30 \mathrm{eV}$. The same characteristic, although less pronounced, is true for feature $F$.

In order to clarify whether this is due to inaccuracies in the band calculations or to shortcomings of the freeelectron final-state approximation, we now turn to more realistic final states derived from an empirical pseudopotential method calculation, where the local potential fitted to optical data used by Cohen and Bergstresser ${ }^{24}$ was applied, taking into account 137 reciprocal-lattice vectors. Figure 5 shows the EPM band structure (above the VBM) and the ETBM bands (below the VBM) along the $\Gamma-\Delta-X$ line in comparison with the free-electron final-state parabola.

The resulting structure plot is shown in Fig. 8 together with the experimental values; the same symbols as in Fig. 6 are used. Symmetry selection rules are not taken into account. As can be seen immediately, the correspondence between experiment and theory is nearly the same as in Fig. 6 for the dispersing features, and in this case no fit parameter like $\left|V_{0}\right|$ is used in order to get a good equivalence. The majority of peaks of $B$ is not very close to theoretical curves; here a better agreement was achieved using free-electron-like final states. Here we mention that the theoretical band gaps in the final states will be closed if a finite damping is introduced in the EPM calculations. Thus structure curves obtained from 


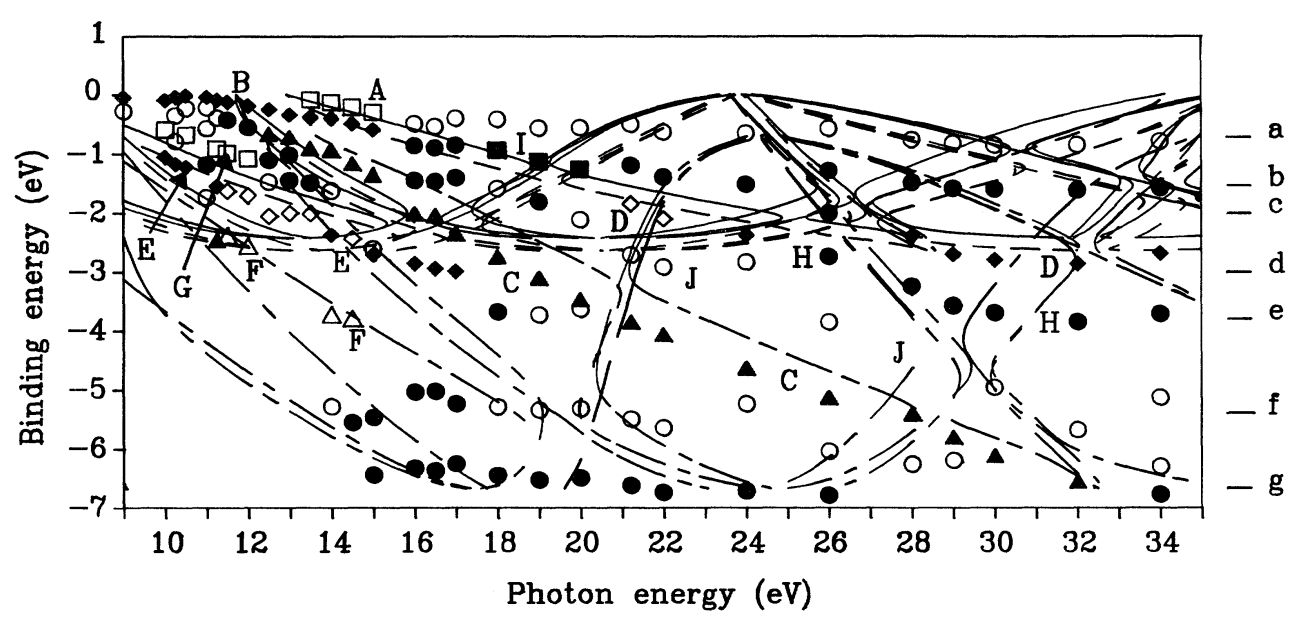

FIG 8. Same as Fig. 6, but with theoretical structure curves resulting from calculated transitions into the pseudopotential final states shown in Fig. 5.

the EPM bands would also fit the emissions of $B$. The most striking facts are the following ones: At first, we find a good correspondence between the high-energy ends of structure curves from the $\Gamma_{7} X_{6}$ band to the emissions of $g$ as well in binding energy as in the photon-energy range, i.e., nearly no emissions at about $-6.8 \mathrm{eV}$ are observed in the photon-energy range from 27 to $31 \mathrm{eV}$, as predicted by theory. Therefore, the principal behavior of feature $g$ should be mainly described by $\mathbf{k}$-conserving bulk transitions. However, the question arises as to why none of the structure curves that attain the $X_{3}$ point energy in the photon energy range from about 14 to $27 \mathrm{eV}$ can be traced in experiment for other binding energies than that of the critical point. Therefore, the emissions are probably caused by other effects, as will be discussed in Sec. III C.

Secondly, with EPM final states the same deviations between the emissions of $C$ and the calculated curves for the $\Gamma_{7} X_{6}$ band are observed as in the case of freeelectron-like final states. At feature $F$ the effect is smaller, but still observable. Even within this refined analysis remain differences between the experimentally observed and the theoretically predicted transitions. But no final decision is possible, whether this is attributable to inaccuracies of the valence- or of the conduction-band structure. While the emissions $A, D, E, F, G$, and $I$ are explained in the same manner as in the structure plot with free-electron-like final states, there is no correspondence between other experimentally observed structures $(J, H$, and the nondispersing features $a-f$ ) and theoretical structure curves.

So far, apart from the correspondence of the peaks of $g$ to parts of theoretical curves, no further significant improvements are accomplished by using EPM final states for the interpretation of structure plots, showing that the free-electron picture in the conduction bands works well. It will be shown in Sec. III C that EPM calculations are very useful in interpreting CIS results.

\section{Interpretation of the CFS results}

In the following we show the results from constantfinal-state spectroscopy measurements in normal emission. This technique described by Lapeyre et al. ${ }^{25,26}$ is a modification of the ARPES method using tunable photon energies from synchrotron radiation. In this mode, in a spectrum the electron detector is kept at fixed positions and fixed electron detection energy, while the photon energy is swept. For instance, in normal emission, assuming free-electron-like final states, one can conclude from Eq. (1) that all peaks observed in a spectrum belong to the same value of $k_{\perp}$, since they have the same kinetic energy. The binding energy of a peak is given by the difference of the kinetic energy and the photon energy and can be related to the VBM if the energetic position of the VBM is known. That way, additional valence-band information can be obtained.

In Fig. 9, some of the measured CFS spectra are shown. The peaks marked by open and closed circles are associated with usual interband transitions, while those marked by rhombs and connected by lines are discussed in Sec. III D. The latter are due to the Ga3d exciton decay process. A band structure obtained from the spectra using Eq. (1) is given in Fig. 10 in comparison with the same theoretical calculation as in Fig. 4. The same value of $\left|V_{0}\right|$ is taken as above, and the energy position of the VBM obtained from the EDC measurements was used. The latter is possible since CFS and EDC spectra were taken at the same samples and under the same conditions.

Apart from some other peaks, which are not connected by lines in Fig. 9, structures labeled $a^{\prime}, g^{\prime}, C^{\prime}, J^{\prime}, W^{\prime}-Z^{\prime}$ are found. While $a^{\prime}, g^{\prime}, C^{\prime}$, and $J^{\prime}$ are easily identified as being associated with the corresponding unprimed transitions in the normal-emission EDC's, the emissions $W^{\prime}-Z^{\prime}$ need additional comment: In order to achieve a good correspondence between the results from EDC and 


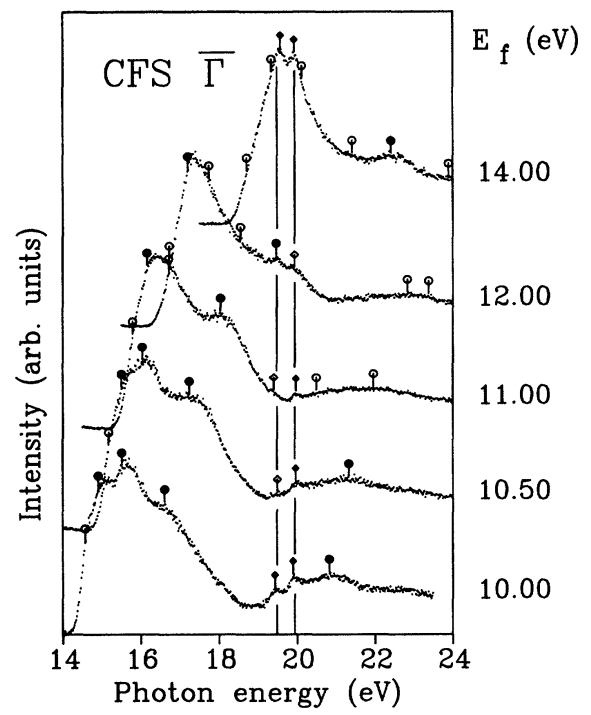

FIG. 9. Constant-final-state spectra in normal emission for different final-state energies plotted on the photon-energy scale. While the peaks marked by circles are due to usual interband transitions, the structures marked by rhombs lying at fixed photon energies are caused by resonance enhancement from the Ga3 $d$ core exciton decay.

CFS, $W^{\prime}$ has to be related to $A, Y^{\prime}$ to $I$, and $X^{\prime}$ to $B$. Therefore, $W^{\prime}$ and $Y^{\prime}$ would be due to excitations from the $\Gamma_{8} X_{7}$ valence band (band 1 in Fig. 10) and $X^{\prime}$ to the $\Gamma_{8} X_{6}$ band (band 2), leaving $Z^{\prime}$ unexplained in the simple evaluation method used in Fig. 10. Even if a to some extent better agreement between theory and experiment is achieved by relating $X^{\prime}$ and $Y^{\prime}$ to the $\Gamma_{8} X_{7}$ and $Z^{\prime}$ to the $\Gamma_{8} X_{6}$ band, this explanation would be quite unlikely, since then the spin-orbit splitting of the uppermost valence bands would become up to about $1 \mathrm{eV}$, i.e., about four times greater than the typical splitting calculated by Chelikowsky and Cohen (e.g., the difference between the

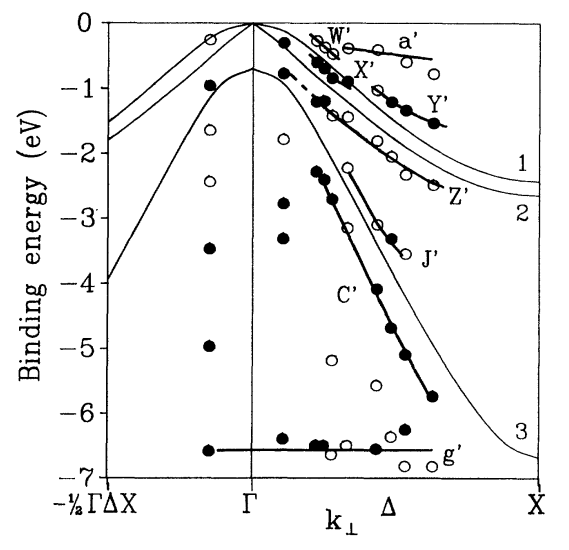

FIG. 10. Experimental band structure along $\Gamma-\Delta-X$ obtained from normal-emission constant-final-state (CFS) spectra using Eq. (1). Closed circles indicate strong, open circles weaker structures. The heavy lines assigned by letters mark experimentally observed structures, the thin lines are the same as in Fig. 4.
$X_{8}$ and $X_{6}$ critical points: $\left.0.24 \mathrm{eV}\right){ }^{15}$ Using the same structure plots as discussed above, but with the experimental results from the CFS measurements, feature $Z^{\prime}$ may be explained by backfolding or surface umklapp transitions because it fits well with some theoretical curves of these plots.

\section{Conclusions from normal-emission measurements}

From the analysis of all normal-emission data the following conclusions can be drawn: The most dispersive structures in the ARPES spectra can be explained by transitions from calculated valence bands into freeelectron-like final states. The disorder of the surface observed in LEED and RHEED has evidently no strong influence on the peak positions of the spectra, but may cause a broadening of the structures observed. Some of the emissions are probably caused by backfolding with different reciprocal surface lattice vectors of the reconstructed surface.

The band-structure calculations for $\mathrm{GaSb}(001)$ does not fit so well to the experimental results as in the case of $\mathrm{GaAs}(001){ }^{7}$ Although the calculated value of the binding energy of the $X_{3}$ critical point is in very good agreement with experiment, the theoretical $\Gamma_{7} X_{6}$ band has a lower binding energy than experimentally observed in its medium part along $\Gamma-\Delta-X$. This effect is observed as well in the free-electron-like final-state approximation as with the even more realistic EPM conduction bands. The same trend is observed in the case of $\mathrm{GaSb}(110){ }^{27}$ The agreement between theory and experiment is better for the $\Gamma_{8} X_{7}$ and the $\Gamma_{8} X_{6}$ bands. With a higher value for the inner potential as chosen in Ref. 18, a better fit would be achieved for the $\Gamma_{7} X_{6}$ band, but the symmetry around the $\Gamma$ point would become worse.

Most results are confirmed by CFS measurements, nevertheless, an exact association of theory, EDC, and CFS results is sometimes difficult for the uppermost valence bands mainly due to the high number of peaks and shoulders observed in this energy range. Nearly all nondispersing or only weakly dispersing features are not consistently explainable as due to emissions from bulk bands. In the next section it will be shown, that most of them are very likely surface-derived states. The energies of the structures $a, b$, and $g$ are quite similar to those of nondispersive emissions in Ref. 18, while the agreement is not so good for the other states, perhaps indicating different reconstructions.

\section{B. Off-normal emission and surface band structure}

In normal-emission spectra we have found about seven states showing no or nearly no dispersion. Since surfacederived bands should not disperse with $k_{1}$, these features are candidates for surface states or resonances, but may be caused by other effects such as, e.g., backfolding of bulk bands with reciprocal surface lattice vectors or nondirect transitions. In order to further investigate the nature of these nondispersive features, spectra in off-normal emission were taken along the lines $\bar{\Gamma} \bar{J}, \bar{\Gamma} \bar{J}^{\prime}$, and $\bar{\Gamma} \bar{K}$ of high symmetry of the ideal $(1 \times 1)$ surface (see Fig. 2$)$ in 
order to also study the dispersion parallel to the surface. As an example, in Fig. 11 a series of spectra taken with He I radiation along the $\bar{\Gamma} \bar{K}$ line of the ideal surface for different polar angles $\vartheta$ is shown. The binding energy is related to the VBM, which was determined with synchrotron radiation as described in Sec. III A. The structures connected by lines and labeled $S_{1}-S_{7}$ will be shown to be surface states or resonances due to their dispersion behavior and their energy positions; nearly all other features are bulk derived. Before turning to the discussion of the surface band structure obtained from the spectra, the methods used for separation of bulk and surface emissions will be described in the next subsection.

\section{Separation of bulk and surface emissions}

In general, there are different ways to separate bulk from surface emissions in the spectra: At first, the results of a normal-emission series can be compared with those of off-normal emission. If an observed band has no dispersion in $k_{\perp}$, but follows the periodicity of the ideal or a reconstructed surface along $k_{\|}$, it should be a surface state or resonance. A backfolded bulk band may also show the periodicity of the surface in off-normal emission, but should normally be dispersive along $k_{\perp}$.

A second criterion for surface states is their sensitivity to adsorbates. Therefore, one can compare spectra before and after exposition of the sample to a defined quantity of an adsorbate such as, e.g., hydrogen, oxygen, or water. While bulk emissions are only damped due to the higher

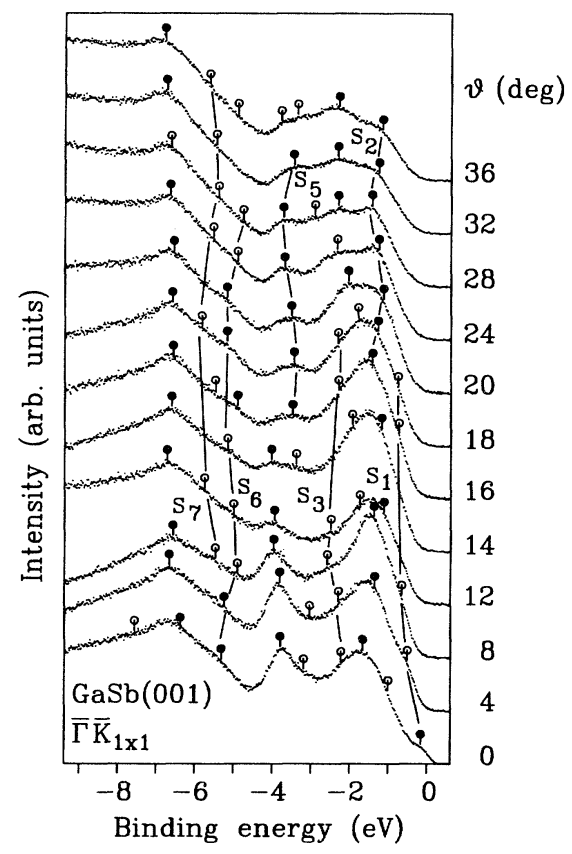

FIG. 11. Angle-resolved photoemission spectra taken with He I energy along the high-symmetry line $\bar{\Gamma} \bar{K}$ of the surface for different polar angles $\vartheta$. The binding energy is related to the valence-band maximum determined from the normal-emission results. The structures marked by $S_{1}-S_{7}$ are due to surface bands, nearly all other emissions not connected by lines to emissions from bulk bands. escape depth of electrons, emissions from surface states should disappear with increasing coverage of the surface. The disadvantage of this method is that the adsorbate may change the surface reconstruction, ${ }^{28}$ leading to a more complex change in the shape of the spectra. Even bulk peaks may be shifted as a consequence of band bending. Therefore, this method was not used here.

A third more theoretically based method is the following: If a bulk band structure $E_{b}(\mathbf{k})$ is given for the entire Brillouin zone, the emissions from these bands in the photoemission process can be calculated for a given photon energy $h v$, assuming $\mathbf{k}$-conserving transitions, since transitions are only possible for $E_{b, f}(\mathbf{k})-E_{b, i}(\mathbf{k})=h v$, where $E_{b, f}(\mathbf{k})$ is the final- and $E_{b, i}(\mathbf{k})$ is the initial-state energy. Transitions at these points can but must not occur as a consequence of selection rules. This method will be described briefly: Given a series of spectra taken along a distinct line of the surface, e.g., a line of high symmetry, for all observed states the wave-vector component parallel to the surface can only lie on this line, i.e., $k_{\|}=\lambda \mathrm{e}$, where $\mathrm{e}$ is a unit vector with the direction of this line and $\lambda$ is a real number. Thus only bulk band transitions can occur, which comply with $E_{b, f}\left(\lambda \mathbf{e}, k_{\perp}\right)$ $-E_{b, i}\left(\lambda \mathrm{e}, k_{\perp}\right)=h v$, whereas in principle $k_{\perp}$ is not restricted, but due to the periodicity of the bulk bands only the range from $k_{\perp}=0$ to $k_{\perp}=4 \pi / a=2 k_{\Gamma \Delta X \Delta \Gamma}$ must be considered. The situation is sketched in Fig. 12(a) for one initial- and one final-state band. Here we have chosen $\mathbf{k}_{\|}$ along the line $\bar{\Gamma} \bar{K}$; the valence band is identical with band 3 of Fig. 4, while the conduction band is a freeelectron final state with $\mathrm{g}_{\|}=0$ and $G_{\perp}=4 \pi / a$ as used in the previous section. For clarity, only the range from $k_{1}=0$ to $k_{\perp}=2 \pi / a$ is shown. The dashed-dotted lines at the bands mark the position, where transitions occur for a given photon energy, here $21.22 \mathrm{eV}$ (He I energy), i.e., where the spacing between the bands is identical with the photon energy. If now these energy positions $E_{b, i}\left(\lambda \mathrm{e}, k_{\perp}\right)=E_{\mathrm{tr}}\left(k_{\|}, k_{\perp}\right)$, at which the transitions occur [e.g., at $k_{\|}=k_{\|, 1}$ as shown in Fig. 12(a)], are projected onto the plane generated by the binding-energy scale and the wave-vector component parallel to the surface, i.e., $k_{\perp}$ is "omitted," then a one-dimensional function $E_{b, i}\left(k_{\|}\right)$ is obtained, which shows the positions where emissions from bulk bands should be seen in an experimental band structure $E_{b}\left(k_{\|}\right)$obtained from an off-normal EDC series. Such a line is plotted in Fig. 12(b). In the plots shown below, we have used the ETBM band structure described in Sec. III A for the valence bands and only those free-electron-like bands described by

$$
E_{b, F}\left(\mathbf{k}_{\|}, \mathrm{k}_{\perp}\right)=\left(\hbar^{2} / 2 \mathrm{~m}\right)\left[\left(\mathrm{k}_{\perp}+\mathrm{G}_{\perp}\right)^{2}+\left(\mathbf{k}_{\|}+\mathrm{G}_{\|}\right)^{2}\right]-\left|\mathrm{E}_{0}\right|,
$$

with $G_{\perp}=0,4 \pi / a, 8 \pi / a, \ldots$, and $G_{\|}=0$ and $\left|E_{0}\right|=5.7$ $\mathrm{eV}$ as determined from normal-emission results. As was shown in Sec. III A these states are the most important ones involved in transitions for photon energies around the energy of $\mathrm{He} \mathrm{I}$ radiation at $21.22 \mathrm{eV}$ used here. If more realistic EPM final states would be applied, this would result in a very large number of transition curves 
leading to quite congested plots, therefore only freeelectron bands were considered.

In the following we will show that this method is a useful tool to separate experimentally bulk from surface states. However, there may remain some problems due to surface umklapp effects or to transitions into final-state bands not included in Eq. (3) and also to inaccuracies in the band-structure calculations. A completely unambiguous identification should combine all of the methods to separate bulk from surface states described above.

\section{Off-normal-emission results}

Turning now to the discussion of the experimental results, we start with a band structure along the $\bar{\Gamma} \bar{K}$ line obtained from the spectra shown in Fig. 11 by the wellknown equation $k_{\|}=\left[\left(2 m / \hbar^{2}\right)\left(E_{\mathrm{kin}} \sin ^{2} \vartheta\right)\right]^{1 / 2}$. The resulting plot with experimental and theoretical data calculated as outlined in Sec. III B 1 is shown in Fig. 13.

Comparing experimental and theoretical results, a very good correspondence is found between the dispersion of observed distinct emissions and the predicted transitions from the uppermost pair of bulk valence bands. These points are therefore interpreted as associated with those
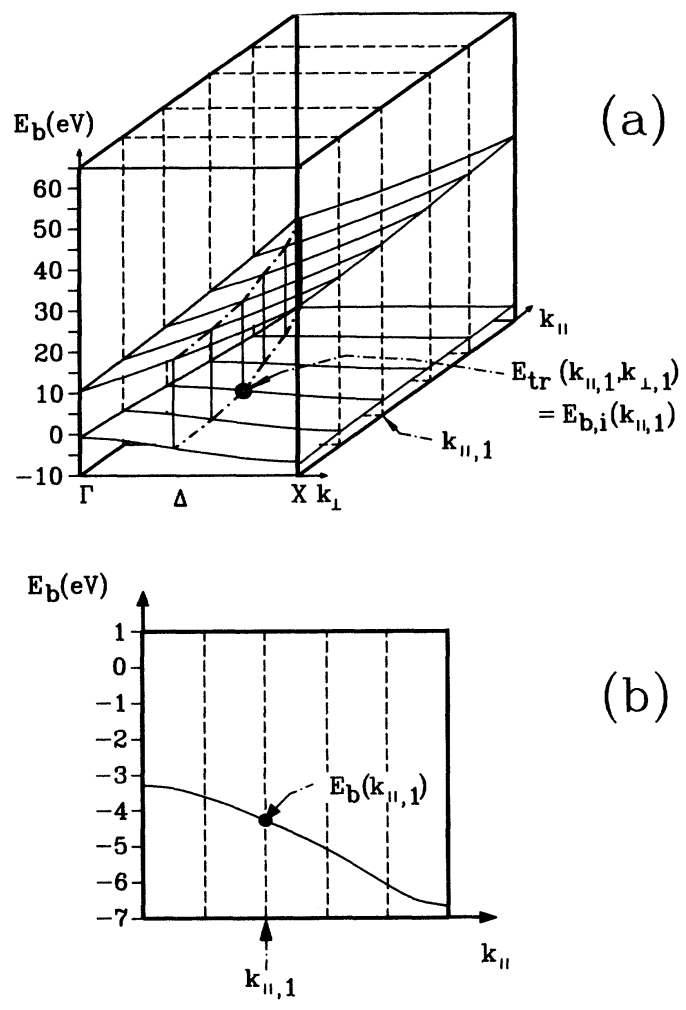

FIG. 12. (a) Possible transitions between a bulk valence band and a conduction band in a photoemission experiment with constant photon energy along a line of high symmetry of the surface. (b) Line of the initial-state energies from transitions from (a), projected on the plane of the binding energy axis and the $k_{\|}$ axis. At such lines, emissions from bulk bands can occur in experimental band structures. For further detail see text. bulk states. Almost no emissions are found corresponding to the calculations of the second pair of valence bands, in accordance with normal-emission results, where emissions from the $\Gamma_{8} X_{6}$ band have only low intensities for photon energies around $20 \mathrm{eV}$. For smaller values of $k_{\|}$up to about $\frac{1}{3} k_{\bar{\Gamma} \bar{K}}$, there is also a strong emission lying below the calculated positions for the split-off valence bands. These peaks are interpreted as caused by those bulk states, because from normal emission it was concluded that the experimentally observed $\Gamma_{7} X_{6}$ band has higher binding energies than the calculated one for the most part.

Nearly all the remaining peaks apart from the feature at about -6.6-eV binding energy can be connected by heavy lines, which show two times $\left(S_{1}\right)$ or four times $\left(S_{2}, S_{3}, S_{5}-S_{7}\right)$ the periodicity of the ideal surface, which in reciprocal space is periodic only on $\bar{\Gamma} \bar{K} \bar{\Gamma}$. At the right side of Fig. 13, the positions of the nondispersing features of the normal-emission measurements are marked by bars and labeled by the same lower-case letters as in Sec. III A. Although in general the number and approximately also the energy positions of these states accord to those of the emissions $S_{1}-S_{7}$ for $k_{\|}=0$, no perfect agreement is found. This may be caused mainly by polarization and matrix-element effects: Some of the states $\left(S_{2}, S_{5}\right.$, and $\left.S_{7}\right)$ cannot be observed in normal emission for the photon energy of $21.22 \mathrm{eV}$, and also at other photon energies the intensity of the emissions is often quite weak in the normal-emission spectra, so that the determination of the peak position is not very accurate for these states. Furthermore, surface resonances may show a small dispersion with $k_{\perp}$.

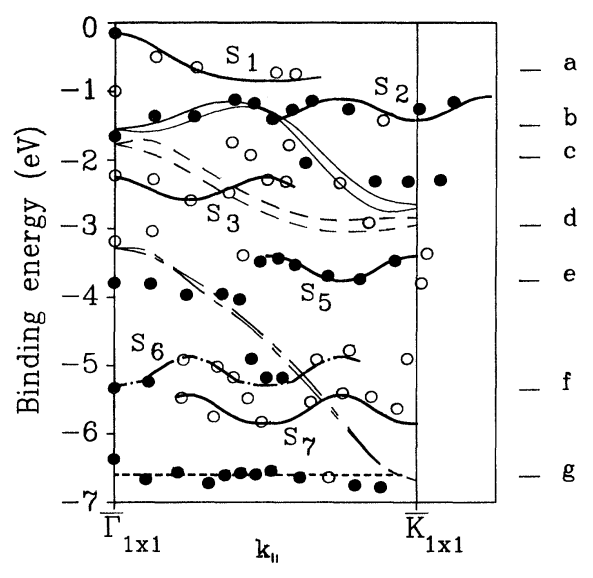

FIG. 13. Experimental band structure along $\bar{\Gamma} \bar{K}$ obtained from the spectra shown in Fig. 11 using Eq. (4). The same lines and symbols are applied as in Fig. 11. The emissions at -6.6 $\mathrm{eV}$ close to the $X_{3}$ critical point are connected by a dashed line. The theoretically predicted positions of bulk emissions in the free-electron final-state approximation for $\mathrm{He}$ I energy are also shown: thin solid lines refer to the uppermost pair of bulk valence bands, thin dashed lines to the second, and the thin dashed-broken lines to the third pair. The bars at the right side of the plot labeled $a-g$ mark the energy positions of nondispersing states from normal-emission spectra. 
Nevertheless, $S_{1}-S_{7}$ are interpreted as surface states or resonances, since they are away from predicted emissions from bulk bands (for transition curves from other bulk bands see below), have the periodicity of a reconstructed surface, and can at least in principle be connected to nondispersive states in normal emission and therefore should not result from backfolding effects of bulk states. The nondispersing feature at about $-6.6 \mathrm{eV}$, marked by a small dashed line has exactly the same energy as structure $g$ of the normal-emission measurements. This structure can be bulk or surface related, as discussed in detail in Sec. III C.

In Fig. 14, the resulting band structure is given for the $\bar{\Gamma} \bar{J}^{\prime} \bar{\Gamma}$ line. The same symbols are used as in Fig. 13; the closed and open rhombs mark the results of a second investigation at another measurement geometry with the sample turned by $90^{\circ}$ around its normal axis with respect to the A vector of the incident light. As can be seen immediately, strong emissions are found in good agreement with the calculated transitions from the uppermost pair of valence bands, and a strong feature about $0.5 \mathrm{eV}$ below the predicted positions of emissions from the third pair of bands is observed. These results are in good accordance with that from the $\bar{\Gamma} \bar{K}$ line. Apart from these features, other emissions are found that cannot be related to the bulk transitions discussed here.

Some of the peaks show no dispersion or less dispersion, especially those at $-2.2,-3.8$, and $-6.6 \mathrm{eV}$. Since they are close in energy to the critical points $X_{5}$, $\Sigma_{1 \mathrm{~min}}$ and $X_{3}$ lying at calculated energies ${ }^{15}$ of -2.5 , -3.6 , and $-6.8 \mathrm{eV}$, respectively, they cannot be directly attributed to emissions from surface states. Eventually the structure at $-2.2 \mathrm{eV}$ may be surface derived, because the surface state $S_{3}$ is found in the same energy range along $\bar{\Gamma} \bar{K}$. The structure at $-6.6 \mathrm{eV}$ is discussed in Sec. III C. In contrast, the structures marked by $S_{1}, S_{1}^{\prime}, S_{4}$, $S_{6}$, and $S_{7}$ show more or less distinct a surface periodicity. While for smaller values of $k_{\|}$up to about $k_{\bar{\Gamma} \bar{J}}$, no other surface state than $S_{1}$ is observed, which shows the simple periodicity of the ideal surface, for higher values of $k_{\|}$we find a doubled periodicity assigned by $S_{1}^{\prime}$. A

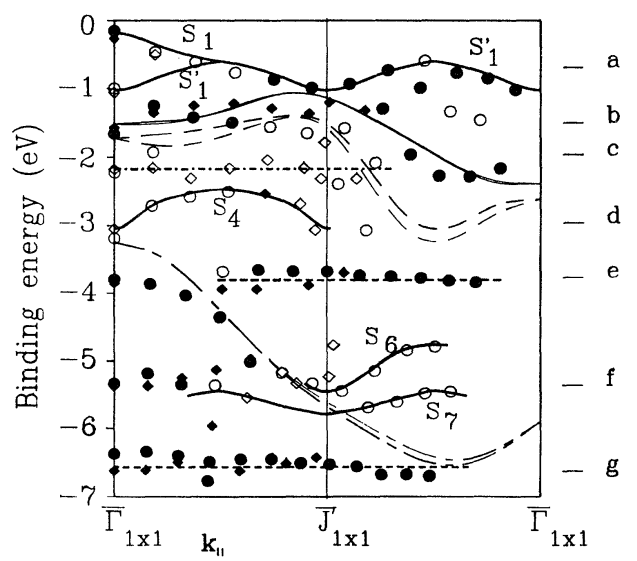

FIG. 14. Same as Fig. 13 for the high-symmetry direction $\bar{\Gamma} \bar{J}^{\prime} \bar{\Gamma}$ of the surface. clear separation between the two states is not possible, since $S_{1}$ cannot be observed over the entire SBZ. The other bands $S_{4}, S_{6}$, and $S_{7}$ are only weak in intensity and are not observable along the whole line $\bar{\Gamma} \bar{J}^{\prime} \bar{\Gamma}$, but are most probably true surface-induced states, because surface bands at the same energies were also found along $\bar{\Gamma} \bar{K}$, though it is not clear whether $S_{4}$ and $S_{5}$ are caused by the same surface band or not. The stronger peaks for lower values of $k_{\|}$close in energy to $S_{6}$ and $S_{7}$ cannot be attributed to these states in an unambiguous way.

Here we mention that also transition curves from bulk emissions were calculated for free-electron final states folded back with reciprocal bulk lattice vectors with nonvanishing $\mathbf{G}_{\|}$. In the $\bar{\Gamma} \bar{J}^{\prime} \bar{\Gamma}$ direction, $S_{1}^{\prime}$ and also parts of $S_{6}$ and $S_{7}$ are lying close to some parts of the calculated curves; $S_{1}^{\prime}$ especially fits quite well to curves belonging to transitions from the uppermost pair of bulk valence bands into those final states with $\mathbf{G}=[\overline{1} 1 \overline{3}]+n[002]$ and $\mathbf{G}=[\overline{2} 0 \overline{2}]+m[002]$ (in units of $2 \pi / a$ in the simple cubic lattice with $n, m$ natural non-negative numbers). Nevertheless, $S_{1}, S_{1}^{\prime}, S_{6}$, and $S_{7}$ are very likely surface bands, since firstly, in normal-emission structure plots, no distinct emissions belonging to those final states were observed in experiment for the photon-energy range around $21.22 \mathrm{eV}$, and secondly, they are in good agreement with surface states along $\bar{\Gamma} \bar{K}$, where no correspondence between $S_{1}, S_{2}, S_{5}-S_{7}$ and any bulk transition curve was found. In this direction only $S_{3}$ is lying in the range of calculated curves, but this may be fortuitous due to the high number of transition curves at this binding energy. Furthermore, if the emissions $S_{1}$ and $S_{1}^{\prime}$ were bulk related, they should be observed in principle also along $\bar{\Gamma} \bar{J}$ (since unpolarized $\mathrm{He} \mathrm{I}$ radiation is used for excitation), in contrast to the experimental results shown below. So $S_{1}, S_{1}^{\prime}, S_{3}, S_{6}$, and $S_{7}$ are interpreted as surface states, but an enhancement of the spectral intensities associated with emissions from bulk bands cannot be excluded.

Additional CFS measurements were performed at the $\bar{J}^{\prime}$ point. From a series of CFS spectra for different final-state energies the resulting band structure is obtained from (1) by replacing $\mathbf{g}_{\|}$by $\mathbf{k}_{\|}$. Dispersing and nondispersing features are found in the band structure. One nondispersing feature is as close in energy to $S_{1}$ as it is to the calculated uppermost pair of valence bands, showing nearly no dispersion along $k_{\perp}$ at the $\bar{J}^{\prime}$ point. Another emission is close to the energy of $S_{6}$ at this point.

Finally, the results for the direction $\bar{\Gamma} \bar{J}$ will also be given in Fig. 15. In the case of bulk emissions we find in principle the same results as on the $\bar{\Gamma} \bar{J}^{\prime}$ line but with less dispersing emissions. Apart from $S_{6}$, which has a periodicity twice that of the ideal SBZ along $\bar{\Gamma} \bar{J}$, no dispersing surface bands are observed. The nearly nondispersing states at about -2.2 and $-3.0 \mathrm{eV}$ may be due to surface states or resonances, since they are close in energy to $S_{3}$ and $S_{4}$, while the emissions at about $-3.8 \mathrm{eV}$, which were also observed along $\bar{\Gamma} \bar{J}^{\prime}$, are probably caused by the $\Sigma_{1 \text { min }}$ point.

In Fig. 16, the resulting experimental surface band structure along lines of high symmetry of the ideal sur- 


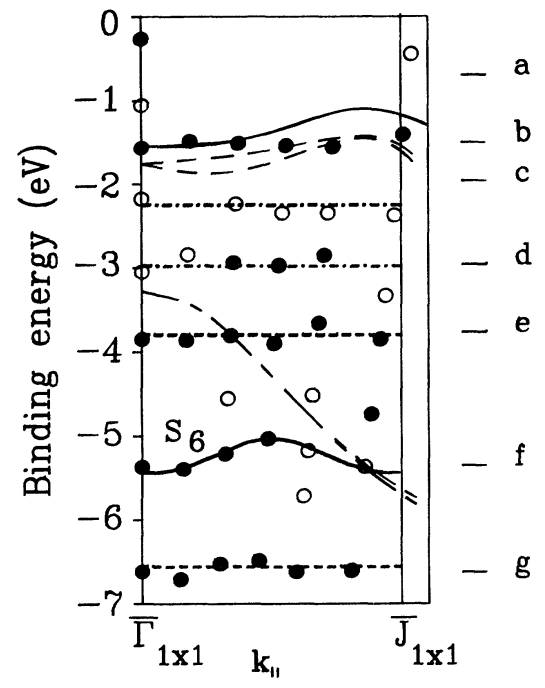

FIG. 15. Same as Fig. 13 for the high-symmetry direction $\bar{\Gamma} \bar{J}$ of the surface. For the assignments of the states marked by heavy dashed and dash-dotted lines see text.

face is plotted. The peak positions were folded back into the ideal SBZ. Along $\bar{\Gamma} \bar{J}$, we have also included the states with nearly no dispersion close in energy to surface states of the $\bar{\Gamma} \bar{J}^{\prime}$ and $\bar{\Gamma} \bar{K}$ lines. The hatched area shows the projected ETBM bulk band structure. The uppermost surface band $S_{1}$, which shows only the periodicity of the unreconstructed surface, starts at about $150 \mathrm{meV}$ below the VBM, and no occupied surface states are found in the fundamental band gap. Furthermore, most of the observed surface-induced bands are lying completely in or at least over a wide range in the hatched area, i.e., they can couple to bulk states and therefore are mainly surface resonances instead of true surface states. Along $\bar{\Gamma} \bar{J}^{\prime}, S_{1}$ is lying close to the edge of the projected bulk band structure, while it may become a true surface state along $\bar{\Gamma} \bar{K}$ for larger values of $k_{\|}$, but the state cannot be traced for $k_{\|} \geq \frac{2}{3} k_{\bar{\Gamma} \bar{K}}$. The surface band $S_{2}$ shows strong emission for $k_{\|} \geq \frac{1}{2} k_{\bar{\Gamma} \bar{K}}$, i.e., beyond the projected bulk band structure, and therefore also seems to be a true surface state. Along $\bar{\Gamma} \bar{K} S_{6}$ and $S_{7}$ also leave the projected bulk band structure for larger values of $k_{\|}$, but they are only weak. Similar to $S_{1}$ along $\bar{\Gamma} \bar{J}^{\prime}, S_{5}$ is lying close to the edge of the projected bulk band structure along $\bar{\Gamma} \bar{K}$.

The most striking fact of the experimental result is the following: From LEED and RHEED experiments, one would expect a $(2 \times 3)$ or $c(2 \times 6)$ periodicity of the electronic surface bands. In the case of $(2 \times 3)$ this is consistent with the results along $\bar{\Gamma} \bar{J}^{\prime}$, where the periodicity of all surface bands apart from $S_{1}$ is twice that of the ideal SBZ. In the case of $c(2 \times 6)$ the unexpected doubling of the periodicity along $\bar{\Gamma} \bar{J}^{\prime} \bar{\Gamma}$ should be accidental, probably caused by the proximity of the $\bar{\Gamma}$ points of the reconstructed surface to the $\bar{J}^{\prime}{ }_{1 \times 1}$ point on the line $\bar{\Gamma} \bar{J}^{\prime} \bar{\Gamma}$ (see Fig. 17), and by some disorder of the surface as observed in LEED and RHEED. Along $\bar{\Gamma} \bar{J}$, in a $(2 \times 3)$ or $c(2 \times 6)$ reconstruction the periodicity of the surface states should be three times that of the ideal surface. The observed bands without any dispersion are in agreement with this condition (as they would be with any other periodicity), but $S_{6}$ is only doubled in periodicity. Even if that were treated as an exception, the interpretation is also problematical for the $\bar{\Gamma} \bar{K}$ line. Here, apart from $S_{1}$, the periodicity of the observed surface emissions seems to be four times that of the ideal SBZ. This result is not expected for a $(2 \times 3)$ or $c(2 \times 6)$ surface, cf. Fig. 2 .
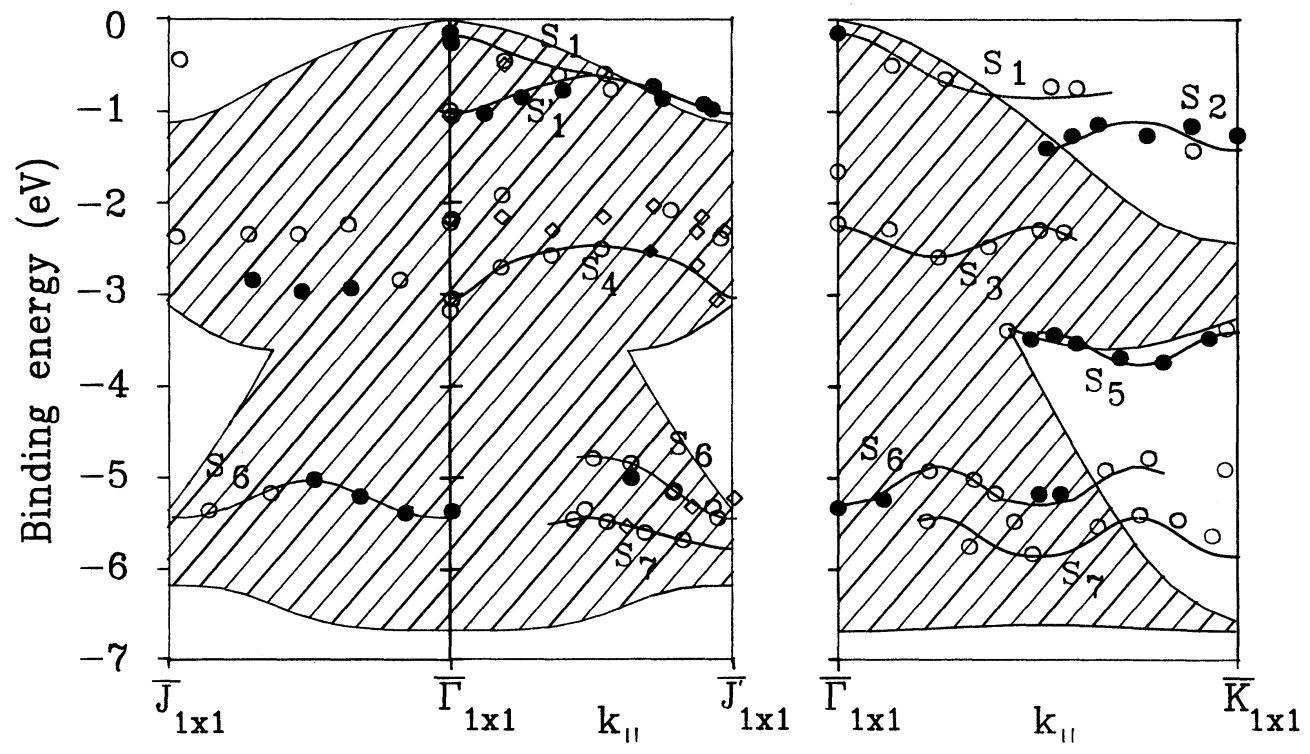

FIG. 16. Surface band structure of $\mathrm{GaSb}(001)$ extracted from the band structures Figs. 13-15. The surface emissions were folded back into the ideal $(1 \times 1)$ surface Brillouin zone. The hatched area shows the projected bulk band structure. 


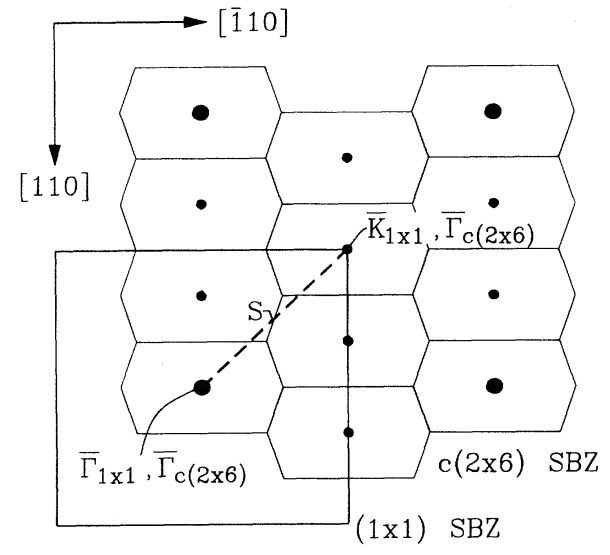

FIG. 17. Possible explanation of the periodicity behavior of the surface states in the periodic zone scheme of the $c(2 \times 6)$ surface reconstruction. The heavy dots present the position of reciprocal-lattice points of the ideal $(1 \times 1)$ surface, the small dots those of the $c(2 \times 6)$ reconstruction. For details see text.

The lowest indexed reconstruction showing a doubling of periodicity along $\bar{\Gamma} \bar{J}^{\prime}$ and a quadrupling along $\bar{\Gamma} \bar{K}$ would be a $c(4 \times 4)$ structure; the doubling along $\bar{\Gamma} \bar{J}$ would also be explained. In this case the geometrical $(2 \times 2)$ or $c(2 \times 6)$ structure would reveal an electronic $c(4 \times 4)$ character. Even if the electronic structure observed is due to the valence-band electrons, while the LEED and RHEED structure is mainly caused by diffraction due to core electrons, such a difference is quite peculiar. In the case of GaAs differences between electronic and geometrical structure have also been reported, ${ }^{4-7}$ but there the electronic periodicity was $(2 \times 1)$ and the periodicity in LEED and RHEED was $(2 \times 4)$ or $c(4 \times 4)$, according to preparation conditions. In reciprocal space, the lattice points of the $(2 \times 1)$ structure are only a subset of that of $(2 \times 4)$ or $c(4 \times 4)$, and this can be a starting point of explanation of this fact. But such an argument would not be applicable to $c(4 \times 4)$ and $(2 \times 3)$ or $c(2 \times 6)$ structures, which are quite different. There may be another explanation for the observed dispersions, if the periodicity of state $S_{6}$ along the line $\bar{\Gamma} \bar{J}$ is assumed to be an exception: If the real surface reconstruction is $c(2 \times 6)$ and not $(2 \times 3)$, then the line $\bar{\Gamma} \bar{K}$ is symmetric around the point $S$ lying at half the distance from $\bar{\Gamma}$ to $\bar{K}$; see Fig. 17. Accordingly, the periodicity of the surface bands is doubled with respect to the ideal surface. This is in agreement with the behavior of $S_{1}$, and the supposed higher symmetry may be only by chance, since the energy positions at $S$ may be nearly the same as at $\bar{\Gamma}$ and $\bar{K}$ and the dispersion around these points can be quite similar.

From the results of this section we can conclude that a separation of bulk and surface emissions was possible investigating the dispersion behavior in normal and offnormal emission and in comparison with theoretical results for the bulk bands. Nevertheless, the observed periodicity of the surface bands is remarkable and calls for theoretical surface-band-structure calculations based on reliable surface structure models, obtainable, e.g., from LEED $I-E$ measurements. A possible surface model for the $c(2 \times 6)$ reconstruction may be deduced from the structure model given in Ref. 18 for the Sb-rich $(1 \times 3)$ reconstruction. Here the main ordering mechanism of the surface should be the dimerization of the uppermost $\mathrm{Sb}$ atoms. If, as in the case of $\mathrm{GaAs}(001)$, tilted dimers are introduced (see, e.g., Ref. 5), the $c(2 \times 6)$ surface can be constructed from the $(1 \times 3)$ model by shifted rows of alternating tilted dimers. It is possible that in the case of Ga-stabilized surfaces, as would be expected for the sputter-annealed samples, the uppermost $\mathrm{Sb}$ atoms have to be replaced by $\mathrm{Ga}$ atoms and vice versa, and the dimers are formed by $\mathrm{Ga}$ atoms. If the second layer is assumed to be a Sb layer as in Ref. 18, then the Ga dimers would have the same direction along the surface as the Ga dangling bonds of an ideal surface. But as in the case of the Sb-stabilized surface, this reconstruction may show also some disorder leading to weak $\frac{1}{2}$-order spots or streaks in LEED and RHEED and probably to the not unique periodicity behavior in the ARPES results for offnormal emission.

\section{Results of constant-initial-state spectroscopy}

Combining the results of normal and off-normal measurements, there remain some problems about the origin of the emissions close in energy to the $X_{3}$ critical point, which are observed in most of the spectra in normal and off-normal emission. They may be interpreted in three different ways:

(a) The emissions are due to direct transitions from the third pair of valence bands into several final states. Considering a theoretical band structure, for nearly any given value of $k_{\|}$along the directions of high symmetry there can be found a value of $k_{\perp}$ with $E_{b, i}\left(k_{\|}, k_{\perp}\right) \approx E_{b}\left(X_{3}\right)$ to within $0.5 \mathrm{eV}$, and electrons close to these points in $\mathbf{k}$ space may be excited for many photon energies due to the great number of final states. The slight dispersion of the initial state can be canceled by effects of backfolding of the third pair of bulk valence bands with reciprocallattice vectors. Furthermore, due to the finite escape depth of the photoelectrons, the $k_{\perp}$ conservation is relaxed.

(b) The peaks are caused by $\mathbf{k}$-conserving transitions from a nearly nondispersive surface state close in energy to the $X_{3}$ critical point into several final states. In this case, excitations with initial-state energy near the $X_{3}$ point would be possible for all photon energies.

(c) Finally, the emissions may be due to a density-ofstates effect, i.e., caused by high non-k-conserving transitions from the $X_{3}$ critical point.

In order to shed light on this problem, we have also performed constant-initial-state spectroscopy measurements. In this modification of the ARPES technique described by Lapeyre et al., ${ }^{25,26}$ in a spectrum the detection energy of the analyzer and the photon energy are simultaneously varied by equal amounts so that their difference remains constant while the photon energy is varied continuously. This way, only excitations from initial states with the same binding energy $E_{b, i}$ into different final states are observed and information mainly about 
the final states can be extracted.

In Fig. 18, a normal-emission CIS spectrum after background subtraction is shown. The photon energy is swept from 15 to $25 \mathrm{eV}$, the kinetic energy (relative to the detector) from 4 to $14 \mathrm{eV}$ resulting in a binding energy of the chosen initial state of $-6.67 \mathrm{eV}$ relative to the VBM. This energy is very close to that of the $X_{3}$ critical point. Due to the low count rate under these conditions the spectral features are quite weak, and a fit was performed after smoothing of the spectrum. The spectrum shows two distinct peaks marked by closed circles and a weaker shoulder marked by an open circle. A satisfying fit with Gaussian peaks was only achieved considering six features; the positions of the three weaker ones are indicated by vertical bars.

Now the observed peak positions for the normalemission spectrum shall be compared with theory. If only strictly k-conserving transitions occur between bulk bands, then at $\mathbf{k}_{\|}=0$ (normal emission) only $k_{\perp} \approx k(X)$ is allowed for transitions, since $E_{b, i} \approx E_{b}\left(X_{3}\right)$ is valid only close to that point along the line $\Gamma-\Delta-X$. In Fig. 19 the distinct peak energies at $\mathbf{k}_{\|}=0$ are plotted as circles at $k_{1}=k(X)$ into a section of the pseudopotential conduction-band structure already used in Sec. III A. The energies of the weak structures obtained by the fit of the CIS spectrum are also presented as crosses. In this figure symmetry selection rules are considered assuming an $s$-like initial state: Only the conduction bands marked by solid lines can be reached from the initial state. All distinct experimental peak positions do not agree well with the calculated values at this point in $\mathbf{k}$ space, where theoretically k-conserving transitions should occur. This fact cannot be attributed only to inaccuracies in the band-structure calculations, since the overall good correspondence between the pseudopotential calculation and the experimental results was proved in Sec. III A. With increasing $k_{\perp}$ relaxation the agreement becomes

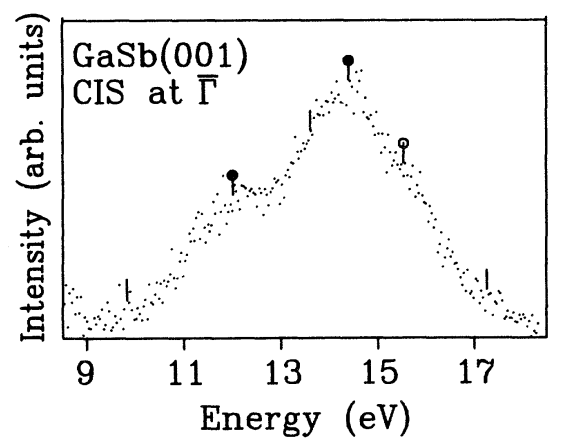

FIG. 18. Constant-initial-state spectrum taken in normal emission. The photon energy is swept from 15 to $25 \mathrm{eV}$, the analyzer detection energy simultaneously from 4 to $14 \mathrm{eV}$ corresponding to an initial-state energy close to the $X_{3}$ critical point of the bulk. The binding energies of the peaks are given by $E_{b, f}=E_{b, i}+h v$ with $E_{b, i}=-6.67 \mathrm{eV}$. The spectrum is shown with subtracted background, closed and open circles give the peak positions of strong and weaker features, respectively. The vertical bars mark positions of peaks needed for a satisfying fit of the spectrum. somewhat better, but from the normal- and off-normalemission results presented above it was observed that no distinct $k_{\perp}$ relaxation must be considered in order to explain the observed emissions. However, a very striking fact is observed if lines are drawn at the final-state energies of the experimentally observed emissions throughout the theoretical band structure along the $\Gamma-\Delta-X$ line shown as horizontal dotted-dashed lines at the positions of the distinct structures and as dotted lines at the energies of the weak features needed for the fit in Fig. 19: The lines belonging to the distinct peaks and shoulders intersect the bulk band structure very close to minima or maxima of calculated bands, i.e., to band edges, marked by small arrows in Fig. 19. The two strong peaks are related to bands, which are allowed for transitions by selection rules. The shoulder at about $15.5 \mathrm{eV}$ also observed can be interpreted as due to the nonideal linear polarization conditions of the incident radiation. The correspondence of the weak features at about 13.5 and $17.2 \mathrm{eV}$ to band edges is not as good as for the stronger ones, but is still quite reasonable. This fact shows that these structures in the spectra are probably not only an artifact of the fit procedure. In the reverse, not all allowed transitions are found in experiment, especially those into the band edges at $k_{\perp}=k_{X}$, but this is probably due to matrix-element effects or to uncertainties in the evaluation of the spectra caused by the broad structures. From this fact we can conclude that an interpretation of the emissions close to the $X_{3}$ critical point in terms of strictly k-conserving bulk transitions without surface effects is

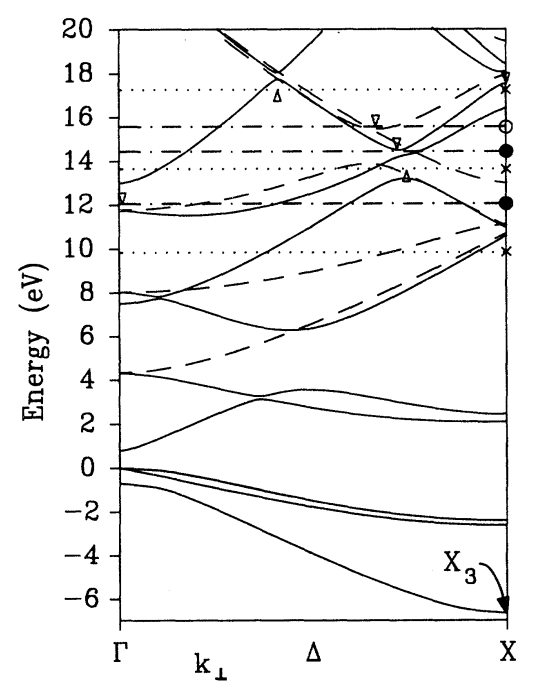

FIG. 19. Theoretical band structure along $\Gamma-\Delta-X$ for $k_{\|}=0$ (same as Fig. 5, but including selection rules for transitions from $s$-like initial states). Also included are the experimental results from the normal-emission spectrum. The circles indicate the points where k-conserving transitions should occur. The crosses present the energetic positions of the very weak features marked by vertical bars in Fig. 18. The horizontal lines at the same energy positions correspond to transitions into band edges marked by small arrows caused by a nondispersing surface state or by effects of backfolding with reciprocal surface lattice vectors. 
not satisfying. But no decision can be made in favor of one of the other mechanisms described above: In the case of a nondispersive surface state close to the criticalpoint energy, i.e., $E_{b, i}\left(\mathbf{k}_{\|}, k_{\perp}\right) \approx E_{b}\left(X_{3}\right)$, excitations are possible at nearly all photon energies, since for any given electron energy $E_{f}$ and the corresponding photon energy $h v \approx E_{f}-E_{b}\left(X_{3}\right)$ used to take the CIS spectrum one finds a final-state band and a value of $k_{\perp}$ along $\Gamma-\Delta-X$, which fulfills $E_{b, f}\left(\mathbf{k}_{\|}=0, k_{\perp}\right)=E_{f}$ and therefore $E_{b, f}\left(\mathbf{k}_{\|}=0, k_{\perp}\right)-E_{b, i}\left(\mathbf{k}_{\|}=0, k_{\perp}\right) \approx E_{f}-E_{b}\left(X_{3}\right) \approx h v$, as is evident from the theoretical band structure. Then the peaks in the spectrum would be caused by the high density of states close to the band edges. The symmetry selection rules are the same, since a surface state in this energy range should be $s$-like also. Almost the same would be true if the initial states are bulk bands folded back with reciprocal surface lattice vectors resulting in very flat bands along the $\Delta$ line, especially if a slight $k_{\perp}$ relaxation of about $5 \%$ of the line $\Gamma-\Delta-X$ and an energy uncertainty of about $0.5 \mathrm{eV}$ is introduced. But non-k-conserving transitions from the critical point $X_{3}$ to critical points of the bulk conduction bands would also explain this behavior. However, the latter is assumed to be very unlikely, since nearly all structures observed in normal and offnormal emission are explainable by almost-k-conserving transitions.

A similar investigation was performed for a series of spectra along the $\bar{\Gamma} \bar{J}^{\prime}$ line of the ideal $(1 \times 1)$ surface. According to the high number of conduction bands apart from the lines of high symmetry in $k_{1}$, here the results are not as distinct as in normal emission, but a reasonable agreement between some of the band edges and experimentally observed transitions is found, while the correspondence between theory and experiment is much worse, if only $\mathbf{k}$-conserving transitions from bulk bands without surface effects are considered.

However, from the CIS results no unique explanation of the emissions close in energy to the $X_{3}$ critical point is found. They may be caused by bulk bands folded back with reciprocal surface lattice vectors or by a nondispersive surface state, but also other reasons cannot be excluded, and probably different effects are superimposed here. For instance, in Sec. III A it was shown that in normal emission the emissions close to $X_{3}$ nearly vanish for photon energies higher than $26 \mathrm{eV}$, as expected for $\mathbf{k}-$ conserving transitions between bulk bands without backfolding. Probably a refinement can be achieved by calculating the transition matrix elements in order to get information about the peak intensities. Nevertheless, the most striking fact is the good correspondence between the energy positions of peaks in the spectra and the calculated band edges, being also a further proof for the accuracy of the pseudopotential calculation in this energy range.

\section{Ga3d core level and excitonic effects}

Constant-final-state measurements were performed for normal emission in order to obtain further information about the Ga3d core level and possible excitonic transitions expected at a photon energy around $20 \mathrm{eV}$. Their appearance could be taken as a hint for an unoccupied surface state close to the conduction-band minimum (CBM). First we discuss the results for the Ga3d core level. In Fig. 20 a CFS spectrum around this level is shown; the final-state energy of the analyzer was chosen as $4 \mathrm{eV}$, while the photon energy was swept from 26.6 to $28.6 \mathrm{eV}$. Because the position of the VBM relative to the analyzer energy scale is known from normal-emission EDC (see Sec. III A), the spectrum can be plotted on a binding-energy scale. Also enclosed in this figure is a fit consisting of two pairs of peaks. The pair of peaks with higher intensity is interpreted as due to the bulk Ga3d core level, the splitting between the $d_{5 / 2}$ and the $d_{3 / 2}$ peaks is about $0.47 \mathrm{eV}$, and the peak height ratio is about 1.54 , in good agreement with the theoretical predicted value of $\left[2 \times\left(\frac{5}{2}\right)+1\right] /\left[2 \times\left(\frac{3}{2}\right)+1\right]=1.5$. The binding energy of the $d_{5 / 2}$ level is $-18.87 \pm 0.1 \mathrm{eV}$. In comparison, Eastman et al. ${ }^{29}$ have found $E_{b}=-18.70 \mathrm{eV}$ and a spinorbit splitting of $0.43 \mathrm{eV}$ at cleaved $\mathrm{GaSb}(110)$ surfaces; Franklin et al. ${ }^{18}$ report $E_{b}=-18.75 \mathrm{eV}$ (if related to the VBM) and a splitting of $0.45 \mathrm{eV}$.

Furthermore, there is a smaller pair of peaks shifted by about $0.20 \mathrm{eV}$ to higher binding energies relative to the bulk pair. Its spin-orbit splitting is $0.49 \mathrm{eV}$ and the peak height ratio is 1.81 . This pair seems to be caused by the changed binding configuration at the surface; the same effect is observed at the MBE prepared $\mathrm{GaSb}(001)$ surface $^{18}$ as well as at cleaved surfaces of $\mathrm{GaSb}(110)$ (Ref. 29) and at GaAs(001) (e.g., see van der Veen et al. for the As $3 d$ core level in Ref. 30). The higher intensity of the spectrum in the energy range of about $-18.4 \mathrm{eV}$ is not explained by the fit. It may be due to a second pair of surface peaks, as a consequence of $\mathrm{Ga}$ in different binding configurations at the reconstructed surface, or to small amounts of pure $\mathrm{Ga}$ at the surface. ${ }^{18}$ But a fit with a total of six peaks is very difficult and the analysis is not very satisfactory. Here we remark that the results for the $\mathrm{Ga} 3 d$ core level are in reasonable agreement with the ob-

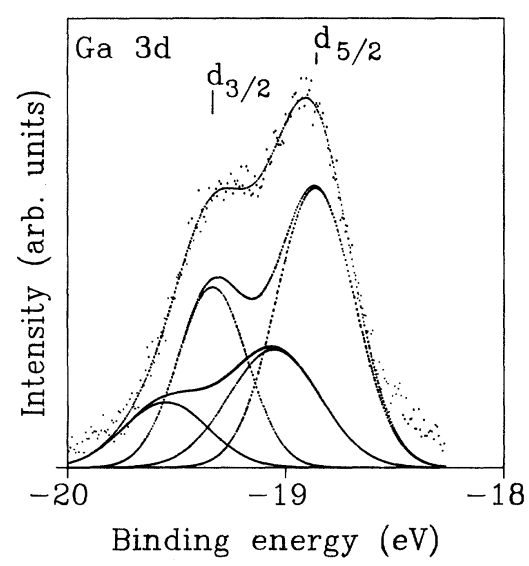

FIG. 20. Constant-final-state spectrum in normal emission of the Ga3 $d$ core level including a fit with two pairs of peaks. The peaks with higher intensity are due to the bulk, the pair with lower intensity shifted to higher binding energies is the surface contribution caused by the changed binding configuration at the surface. 
servations of Franklin et al. ${ }^{18}$ apart from the shift of the surface pair relative to the bulk pair, which was $0.43 \mathrm{eV}$ in Ref. 18. This fact, together with the EDC results for the energy positions of surface states, indicates that the surface reconstruction investigated here is different from that in Ref. 18, probably in a way as discussed at the end of Sec. III B.

Turning now to the discussion of excitonic effects from the Ga3 $d$ core level, in Fig. 9 a series of CFS spectra taken in normal emission for different final-state energies (relative to the analyzer) is shown. In this case, the surface normal, the [110] direction $\bar{\Gamma} \bar{J}$, and the $\mathbf{A}$ vector of the incident synchrotron radiation were lying in the same plane, $\mathbf{A}$ at an angle of about $45^{\circ}$ with the surface. Apart from the structures already explained in Sec. III A, a pair of peaks at about $20-\mathrm{eV}$ photon energy is found marked by rhombs, which remains at the same photon energy for all spectra. Such features are not related to bulk or surface interband transitions. The peaks separated by about $0.46 \mathrm{eV}$, nearly exactly the energy of the spin-orbit splitting of the Ga3d core level, are interpreted as being caused by a resonant-type Ga3d core exciton decay with an excitation energy of $19.48 \pm 0.05 \mathrm{eV}$ and $19.94 \pm 0.05$ $\mathrm{eV}$ for the $d_{5 / 2}$ and $d_{3 / 2}$ core levels, respectively. The nature of this exciton is not completely clear: In analogy to the results from (110) surfaces of different III-V semiconductors, ${ }^{25,31}$ the exciton is assumed to couple to empty $\mathrm{Ga}$ surface states, which is also confirmed by the analysis of its polarization dependence, described below. This would be possible, since a surface treated by IBA should be rich in the components with the lower vapor pressure, i.e., gallium, possibly leading to unoccupied dangling-bond states at the surface. The core levels attributed to the exciton can be those of the surface or those of the bulk, which are shifted by about $0.20 \mathrm{eV}$. If the exciton decay is related to the bulk core levels at $E_{b}=-18.87 \pm 0.1 \mathrm{eV}$ and $E_{b}=-19.34 \pm 0.1 \mathrm{eV}$ for the $d_{5 / 2}$ and $d_{3 / 2}$ levels, respectively, then the exciton level would be located at about $0.61 \pm 0.15 \mathrm{eV}$ above the VBM. Assuming a band gap of $0.70 \mathrm{eV}$ at room temperature following Ref. 32, this would result in a binding energy relative to the conduction-band minimum (CBM) of about $-0.09 \pm 0.15 \mathrm{eV}$. If, on the other hand, the exciton decay is related to the surface core levels, its binding energy would become $-0.29 \pm 0.15 \mathrm{eV}$ relative to CBM. Here the CBM is used as reference level, since to our knowledge the energy position of the Ga-derived unoccupied surface band has not yet been determined for the surface studied here.

Finally, we remark that the exciton decay is quite weak when the sample is rotated by $90^{\circ}$; i.e., $\mathbf{A}$, the surface normal and the [ $\overline{1} 10]$ direction $\bar{\Gamma} \bar{J}^{\prime}$ are lying in the same plane. Therefore, no excitonic effects were found in the CIS spectra discussed in Sec. III C, which were taken for the latter sample orientation. This fact shows the sensitivity of the exciton to polarization effects. If the exciton couples to empty $\mathrm{Ga}$ states, in a very simple picture one would expect some similarity between the character of the excitonic final-state wave function and that of the $\mathrm{Ga}$ dangling bond: If the $\mathrm{Ga}$ dangling bond is assumed to have the same direction for the reconstructed as for the ideal surface, higher intensity of the transition is expected for A parallel to the [110] direction due to a matrix element $\left|\left\langle\psi_{i}|\mathbf{A} \cdot \mathbf{p}| \psi_{f}\right\rangle\right|^{2}$, increasing with decreasing angle between $\mathbf{A}$ and the main direction of the final-state wave function. In order to obtain a better interpretation of this behavior, theoretical calculations for the excitonic wave functions are necessary.

\section{CONCLUSIONS}

We have performed a detailed study of the electronic band structure of $\mathrm{GaSb}(001)$ by angle-resolved photoemission spectroscopy, using systematically three different modes of ARPES: energy-distribution curves, constant-initial, and constant-final-state spectroscopy. In normal-emission EDC's, the most dispersing features with varying photon energy could be explained in a first approximation assuming free-electron-like final-state bands with a common value of $11 \mathrm{eV}$ for $\left|V_{0}\right|$. With this value the best symmetry of the emissions from the $\Gamma_{8} X_{6}$ band around $\Gamma$ is obtained. Backfolding effects with reciprocal bulk and surface lattice vectors are also likely involved in the transitions. Structure plots have been used as an adequate method of comparison between experimental and theoretically predicted results. Refinement of the final-state parabola by a pseudopotential conductionband calculation gives no essential further improvement apart from a partial explanation of the emissions close to the $X_{3}$ critical point. The valence-band-structure calculation used here does not seem to fit equally well to the experimental data as in the case of the low-indexed surfaces of GaAs, but the fundamental behavior is reproduced with good accuracy. The results were also compared with constant-final-state data showing the same trend.

In off-normal emission, a surface band structure was derived by comparison with normal-emission data and by calculating the expected peak positions of bulk transitions in off-normal emission in order to separate these from the surface-related features. Up to seven surface bands were found showing the dispersion of a reconstructed surface. Nevertheless, the periodicity is not fully consistent with the results from LEED and RHEED observations, and calculations of electronic surface band structures for different surface reconstructions of $\mathrm{GaSb}(001)$ are needed.

An unambiguous explanation of the origin of the nondispersive emissions observed in all spectra close in energy to the $X_{3}$ critical point was not achieved even with CIS spectra. They can be due to a surface-derived valence band or to bulk bands folded back with reciprocal surface lattice vectors. The main result of the CIS investigations is the quite good agreement of the experimentally observed peak positions with the energy of band edges in the calculated conduction-band structure, proof of the accuracy of the EPM calculations.

We have also investigated the Ga3d core level consisting of a bulk and a surface spin-orbit pair of emissions shifted by about $0.20 \mathrm{eV}$. In constant-final-state spectra, an intensity enhancement due to the Ga3d exciton decay is observed depending on the polarization conditions. 
This exciton suggests an unoccupied surface state of the very likely Ga-rich surface reconstruction prepared by ion bombardment and annealing.

Altogether, no further improvement of the data interpretations seems possible, if only energy- and momentum-conserving transitions and in some cases slight $k_{\perp}$ relaxation are considered using a one-electron Hamiltonian without calculation of the transition matrix elements. For a refinement of the analysis, calculations of photoemission spectra within the one-step model are needed, which are based on reliable bulk and surface band structures. Even if the EPM band structure is shown to fit well with the results of CIS, the theoretical band structures should rather be replaced by more sophisticated calculations, e.g., self-energy quasiparticle states, which have been successfully applied for other semiconductors. ${ }^{33}$

\section{ACKNOWLEDGMENTS}

This work was supported by the Bundesministerium für Forschung und Technologie (Bonn, Federal Republic of Germany), Project No. 05401 AAI and by the Deutsche Forschungsgemeinschaft (Bonn, Federal Republic of Germany), Project No. Scha 360/2-2. The calculations were performed at the Rechenzentrum der Universität Kiel.
${ }^{*}$ Permanent address: Vakuum-Anlagenbau G.m.b.H. Sandhöhe 11, D-2200 Elmshorn, Federal Republic of Germany.

${ }^{1}$ G. V. Hansson and R.I.G. Uhrberg, Surf. Sci. Rep. 9, 197 (1988), and references cited therein.

${ }^{2}$ G. P. Williams, F. Cerrina, G. J. Lapeyre, J. R. Anderson, R. J. Smith, and J. Hermanson, Phys. Rev. B 34, 5548 (1986).

3J. Henk, W. Schattke, H.-P. Barnscheidt, C. Janowitz, R. Manzke, and M. Skibowski, Phys. Rev. B 39, 13286 (1989).

${ }^{4}$ R. D. Bringans and R. Z. Bachrach, in Proceedings of the 17th International Conference on the Physics of Semiconductors, San Francisco, 1984, edited by W. A. Harrison (Freeman, San Francisco, 1984).

${ }^{5}$ P. K. Larsen, J. F. van der Veen, A. Mazur, J. Pollmann, J. H. Neave, and B. A. Joyce, Phys. Rev. B 26, 3222 (1982).

${ }^{6}$ P. K. Larsen, J. H. Neave, J. F. van der Veen, P. J. Dobson, and B. A. Joyce, Phys. Rev. B 27, 4966 (1983).

${ }^{7}$ J. Olde, G. Mante, H.-P. Barnscheidt, L. Kipp, J.-C. Kuhr, R. Manzke, M. Skibowski, J. Henk, and W. Schattke, Phys. Rev. B 41, 9958 (1990).

${ }^{8}$ L. Kipp, C. Janowitz, G. Mante, H. P. Barnscheidt, J. Olde, R. Manzke, and M. Skibowski, Vacuum 41, 608 (1990).

${ }^{9}$ R. Manzke and M. Skibowski, Phys. Scr. T 31, 87 (1990).

${ }^{10}$ A. Zunger, Phys. Rev. B 22, 959 (1980).

${ }^{11}$ C. Mailhiot, C. B. Duke, and D. J. Chadi, Phys. Rev. B 31, 2213 (1985).

${ }^{12}$ S. Y. Tong, G. Xu, W. Y. Hu, and M. W. Puga, J. Vac. Sci. Technol. B 3, 1076 (1985).

${ }^{13}$ D. J. Chadi, Phys. Rev. Lett. 57, 102 (1986).

${ }^{14} \mathrm{~J}$. Henk and W. Schattke, Solid State Commun. 70, 683 (1989).

15J. R. Chelikowsky and M. L. Cohen, Phys. Rev. B 14, 556 (1976).

${ }^{16}$ T.-C. Chiang and D. E. Eastman, Phys. Rev. B 22, 2940 (1980).

${ }^{17}$ R. Manzke, H.-P. Barnscheidt, C. Janowitz, and M. Ski- bowski, Phys. Rev. Lett. 58, 610 (1987).

${ }^{18}$ G. E. Franklin, D. H. Rich, A. Samsavar, E. S. Hirschhorn, F. M. Leibsle, T. Miller, and T.-C. Chiang, Phys. Rev. B 41, 12619 (1990).

${ }^{19}$ B. A. Joyce, J. H. Neave, P. J. Dobson, and P. K. Larsen, Phys. Rev. B 29, 814 (1984).

${ }^{20}$ Chin-An Chang, R. Ludeke, L. L. Chang, and L. Esaki, Appl. Phys. Lett. 31, 759 (1977).

${ }^{21}$ T. H. Chiu and W. T. Tsang, J. Appl. Phys. 57, 4572 (1985).

${ }^{22} \mathrm{M}$. Lehmann, masters thesis, Universität Kiel, 1988.

${ }^{23}$ From our photoemission work on $\mathrm{GaSb}(110)$ it results that $\left|V_{0}\right|$ is about $14 \mathrm{eV}$ for the $\Gamma-K-X$ emission direction.

${ }^{24}$ M. L. Cohen and T. K. Bergstresser, Phys. Rev. 141, 789 (1966).

${ }^{25}$ G. J. Lapeyre, R. J. Smith, J. Knapp, and J. Anderson, J. Phys. (Paris) Colloq. 39, C4-134 (1978).

${ }^{26}$ G. J. Lapeyre, A. D. Baer, J. Anderson, J. C. Hermanson, J. A. Knapp, and P. L. Gobby, Solid State Commun. 15, 1601 (1974).

${ }^{27}$ H.-P. Barnscheidt, Ph.D. thesis, Universität Kiel, 1989.

${ }^{28}$ R. D. Bringans and R. Z. Bachrach, J. Vac. Sci. Technol. A 1, 676 (1983).

${ }^{29}$ D. E. Eastman, T.-C. Chiang, P. Heimann, and F. J. Himpsel, Phys. Rev. Lett. 45, 656 (1980).

30J. F. van der Veen, P. K. Larsen, J. H. Neave, and B. A. Joyce, Solid State Commun. 49, 659 (1984).

${ }^{31}$ G. J. Lapeyre and J. Anderson, Phys. Rev. Lett. 35, 117 (1975).

${ }^{32}$ G. Harbeke, O. Madelung, and V. Rössler, in Physics of Group $I V$-Elements and III-V-Compounds, edited by O. Madelung, Landolt-Börnstein, New Series, Group III, Vol. 17, Pt. a (Springer-Verlag, Berlin, 1982), p. 258.

${ }^{33}$ M. S. Hybertsen and S. G. Louie, Phys. Rev. B 34, 5390 (1986). 


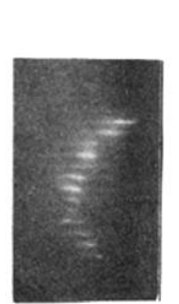

(a)

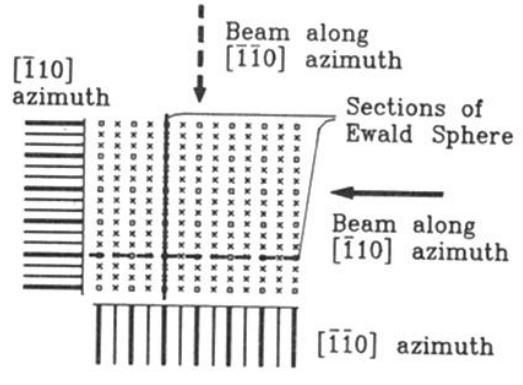

(c)

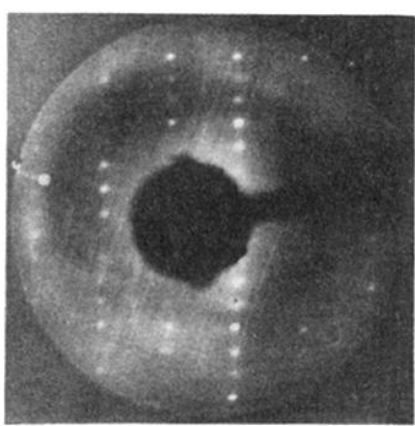

(d)

(b)

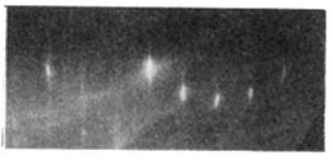

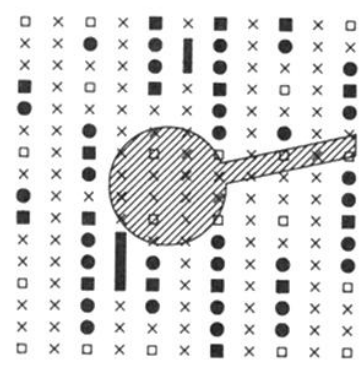

(e)

FIG. 1. (a) and (b): RHEED pattern of $\mathrm{GaSb}(001)-(2 \times 3)$ at $8-\mathrm{keV}$ kinetic energy. In (a) the [ $\overline{1} 10]$ azimuth is shown, (b) is the same for the [ $\overline{1} 10]$ azimuth. In (c) the reciprocal lattice is sketched together with the incident beam direction, sections of the Ewald sphere, and the resulting RHEED pictures. The reciprocal-lattice rods from the ideal $(1 \times 1)$ surface are plotted as squares or heavy lines, those from the reconstructed surface as crosses or thin lines at the left and lower side of (c). (d) LEED pattern of the same reconstruction at 116-eV kinetic energy. In (e) the reciprocal lattice of $(2 \times 3)$ is shown together with the experimental results plotted as closed squares, closed circles or heavy lines: the $\frac{1}{2}$-order reflexes are blurred to lines. The hatched area shows the position of the electron gun. 